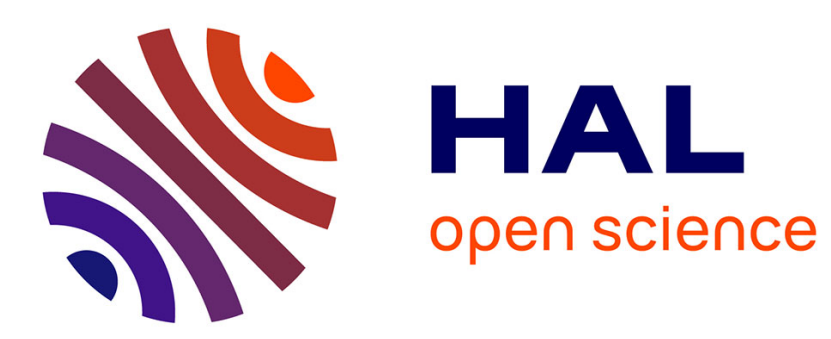

\title{
Pointwise second-order necessary optimality conditions for the Mayer problem with control constraints
}

Hélène Frankowska, Daniela Tonon

\section{To cite this version:}

Hélène Frankowska, Daniela Tonon. Pointwise second-order necessary optimality conditions for the Mayer problem with control constraints. SIAM Journal on Control and Optimization, 2013, 51 (5), pp.3814-3843. 10.1137/130906799 . hal-00918491

\section{HAL Id: hal-00918491 \\ https://hal.science/hal-00918491}

Submitted on 16 Dec 2013

HAL is a multi-disciplinary open access archive for the deposit and dissemination of scientific research documents, whether they are published or not. The documents may come from teaching and research institutions in France or abroad, or from public or private research centers.
L'archive ouverte pluridisciplinaire HAL, est destinée au dépôt et à la diffusion de documents scientifiques de niveau recherche, publiés ou non, émanant des établissements d'enseignement et de recherche français ou étrangers, des laboratoires publics ou privés. 


\title{
POINTWISE SECOND-ORDER NECESSARY OPTIMALITY CONDITIONS FOR THE MAYER PROBLEM WITH CONTROL CONSTRAINTS
}

\author{
HÉLÈNE FRANKOWSKA AND DANIELA TONON
}

\begin{abstract}
This paper is devoted to second order necessary optimality conditions for the Mayer optimal control problem when the control set $U$ is a closed subset of $\mathbb{R}^{m}$. We show that, in the absence of endpoint constraints, if an optimal control $\bar{u}(\cdot)$ is singular and integrable, then for almost every $t$ such that $\bar{u}(t)$ is in the interior of $U$, both the Goh and a generalized LegendreClebsch conditions hold true. Moreover, when the control set is a convex polytope, similar conditions are verified on the tangent subspace to $U$ at $\bar{u}(t)$ for almost all $t$ 's such that $\bar{u}(t)$ lies on the boundary $\partial U$ of $U$. The same conditions are valid also for $U$ having a smooth boundary at every $t$ where $\bar{u}(\cdot)$ is singular, locally Lipschitz and $\bar{u}(t) \in \partial U$.

In the presence of a smooth endpoint constraint, these second order necessary optimality conditions are satisfied whenever the Mayer problem is calm and the maximum principle is abnormal. If it is normal, then analogous results hold true on some smaller subspaces.
\end{abstract}

Key words. Optimal control, second order necessary conditions, endpoint constraint.

AMS Mathematics Subject Classification 2010. Primary 49K15. Secondary 49N10.

\section{INTRODUCTION}

Consider the Mayer optimal control problem

$$
\min \left\{\varphi(x(1)): x(\cdot) \in S_{[0,1]}\left(x_{0}\right), x(1) \in K\right\},
$$

where $\varphi: \mathbb{R}^{n} \rightarrow \mathbb{R}$ is a given cost function, $K$ is a closed subset of $\mathbb{R}^{n}$ and $S_{[0,1]}\left(x_{0}\right)$ is the set of all absolutely continuous solutions of the control system

$$
x^{\prime}(t)=f(t, x(t), u(t)), \quad u(t) \in U \quad \text { for a.e. } t \in[0,1], x(0)=x_{0} .
$$

In the above $U$ is a closed subset of $\mathbb{R}^{m}$ and $f:[0,1] \times \mathbb{R}^{n} \times \mathbb{R}^{m} \rightarrow \mathbb{R}^{n}$ is sufficiently regular. A pair $(x(\cdot), u(\cdot))$, with $x(\cdot)$ absolutely continuous and $u(\cdot)$ measurable, is called a trajectory/control pair if it satisfies $(1.2)$.

The Hamiltonian $\mathcal{H}:[0,1] \times \mathbb{R}^{n} \times \mathbb{R}^{n} \times \mathbb{R}^{m} \rightarrow \mathbb{R}$ and the maximized Hamiltonian $H$ : $[0,1] \times \mathbb{R}^{n} \times \mathbb{R}^{n} \rightarrow \mathbb{R} \cup\{+\infty\}$ associated to the Mayer problem are defined respectively by

$$
\mathcal{H}(t, x, p, u):=\langle p, f(t, x, u)\rangle, \quad H(t, x, p):=\sup _{u \in U} \mathcal{H}(t, x, p, u) .
$$

Consider a trajectory/control pair $(\bar{x}(\cdot), \bar{u}(\cdot))$ which is a strong local minimizer of the above minimization problem. Then the first order necessary optimality conditions are given by the Pontryagin maximum principle in order to select candidates for being optimal trajectory/control pairs among admissible ones.

We start by discussing this problem in the absence of endpoint constraints, i.e. the Mayer problem

$$
\min \left\{\varphi(x(1)): x(\cdot) \in S_{[0,1]}\left(x_{0}\right)\right\}
$$

Date: July 31, 2013.

A short version of this paper announcing results with no proofs is to appear in Proceedings of the 52nd IEEE Conference on Decision and Control, Florence, Italy, December 10-13, 2013. 
Then the Pontryagin maximum principle says that there exists an absolutely continuous function $p:[0,1] \rightarrow \mathbb{R}^{n}$ satisfying the adjoint system

$$
-p^{\prime}(t)=f_{x}(t, \bar{x}(t), \bar{u}(t))^{*} p(t)=\mathcal{H}_{x}(t, \bar{x}(t), p(t), \bar{u}(t)) \text { for a.e. } t \in[0,1],
$$

the maximum principle

$$
\langle p(t), f(t, \bar{x}(t), \bar{u}(t))\rangle=H(t, \bar{x}(t), p(t)) \text { for a.e. } t \in[0,1]
$$

and the transversality condition

$$
-p(1)=\nabla \varphi(\bar{x}(1))
$$

The above maximum principle is normal because the endpoint constraints are absent. As a direct consequence of it, for almost all $t \in[0,1]$ such that the optimal control $\bar{u}(t)$ lies in the interior of the control constraint $U, \mathcal{H}_{u}[t]=0$ and $\mathcal{H}_{u u}[t] \leq 0$. This last inequality is known as the classical Legendre-Clebsch condition. Here and in the following, the abbreviation $[t]$ stands for $(t, \bar{x}(t), p(t), \bar{u}(t))$. When $\mathcal{H}_{u u}[\cdot]=0$ (for instance the affine control systems do have $\mathcal{H}_{u u}=0$ ), the Legendre-Clebsch condition doesn't bring any additional information about optimal trajectory/control pairs, and different pointwise second-order optimality conditions turn out to be necessary in order to deal with this case.

In this paper we derive pointwise second-order necessary optimality conditions for a trajectory/control pair $(\bar{x}(\cdot), \bar{u}(\cdot))$ which is a strong local minimizer and such that $\mathcal{H}_{u u}[t]=0$ a.e. in $\left[t_{1}, t_{2}\right]$ for some $0 \leq t_{1}<t_{2} \leq 1$. We call such $(\bar{x}(\cdot), \bar{u}(\cdot))$ singular. Let us underline that in the literature singular controls may refer to several situations: vanishing switching function as for instance in [22], non surjective endpoint mapping as in [8, while in [17, 20] singularity of an extremal means $\mathcal{H}_{u u}[t]$ not having the full rank. Although most of our results are valid also for weak local minimizers, to simplify their presentation we restrict our attention to strong local minimizers only. The derived second order conditions are a generalization of the conditions introduced by Goh in [15], to the case in which a closed control constraint and a smooth endpoint constraint are present. According to [15], when $U$ is an open hypercube, under suitable regularity hypotheses on $f$, the matrix $\mathcal{H}_{x u}[t] f_{u}[t]$ is symmetric and

$$
R(t):=\frac{1}{2} f_{u}[t]^{*} \mathcal{H}_{x x}[t] f_{u}[t]-\frac{1}{2} \frac{d}{d t} \mathcal{H}_{x u}[t] f_{u}[t]-\mathcal{H}_{x u}[t] f_{x}[t] f_{u}[t]+\frac{1}{2} \mathcal{H}_{x u}[t] \frac{d}{d t} f_{u}[t]
$$

is negative semi-definite for a.e. $t \in\left[t_{1}, t_{2}\right]$. The first property, known in the literature as the Goh condition, in the case of affine control systems reduces to a condition on the Lie brackets of the flux functions, as we recall in Remark 3.2. The second property can be seen as a generalized Legendre-Clebsch condition. Both conditions were proved by applying the classical Legendre-Clebsch condition to a new control problem of linear-quadratic type obtained after a transformation, introduced by Goh in [16, of the following classical second-order optimality condition

$$
0 \geq \int_{0}^{1}\left(\frac{1}{2} y(t)^{*} \mathcal{H}_{x x}[t] y(t)+u(t)^{*} \mathcal{H}_{x u}[t] y(t)+\frac{1}{2} u(t)^{*} \mathcal{H}_{u u}[t] u(t)\right) d t-\frac{1}{2} y(1)^{*} \varphi^{\prime \prime}(\bar{x}(1)) y(1) .
$$

In the above $u(\cdot) \in L^{\infty}\left([0,1] ; \mathbb{R}^{m}\right)$ and $y(\cdot)$ solves the following linear system (variational equation)

$$
\left\{\begin{array}{l}
y^{\prime}(t)=f_{x}[t] y(t)+f_{u}[t] u(t) \\
y(0)=0
\end{array}\right.
$$

The Goh and the generalized Legendre-Clebsch conditions were proved in [15] for optimal trajectory/control pairs for which there exists a regular transformation of $\mathcal{H}_{u u}[\cdot]$ into a matrix valued mapping of the following type

$$
\left[\begin{array}{cc}
\mathcal{R}_{1}[\cdot] & 0 \\
0 & 0
\end{array}\right]
$$


where $\mathcal{R}_{1}[\cdot]$ is a non-singular $(r \times r)$-matrix and $r$ is the rank of $\mathcal{H}_{u u}[\cdot]$, supposed to be constant on $[0,1]$. Though our results are proved only for the case $\mathcal{H}_{u u}[t]=0$ for a.e. $t \in\left[t_{1}, t_{2}\right]$ for some $0 \leq t_{1}<t_{2} \leq 1$, they can be easily generalized to the above situation.

Goh also suggested two possible strategies to deal with closed control constraints. The first one is to replace (1.7) by an equivalent inequality in which control constraints are absent, using Valentine's approach from [21], 7] for piecewise $C^{2}$ trajectory/control pairs. The second one is to use variations of the optimal control which are equal to zero when $\bar{u}(t) \in \partial U$. However, both are in a way weak, since the first one requires too much regularity of the optimal control, while the second one can't be used to recover second-order optimality conditions when $\bar{u}(t) \in \partial U$ almost everywhere.

In this paper we present a different approach, using non-zero variations of the optimal control that remain in the control constraint even when $\bar{u}(\cdot)$ takes values in the boundary of $U$. This is performed using a slightly different second-order optimality condition derived by Hoehener in [18]:

$$
\begin{aligned}
0 \geq & \int_{0}^{1}\left(\mathcal{H}_{u}[t] v(t)+\frac{1}{2} y(t)^{*} \mathcal{H}_{x x}[t] y(t)+u(t)^{*} \mathcal{H}_{x u}[t] y(t)+\frac{1}{2} u(t)^{*} \mathcal{H}_{u u}[t] u(t)\right) d t \\
& -\frac{1}{2} y(1)^{*} \varphi^{\prime \prime}(\bar{x}(1)) y(1)
\end{aligned}
$$

that holds for measurable bounded admissible variations $u(\cdot), v(\cdot)$ such that $\mathcal{H}_{u}[t] u(t)=0$, $u(t) \in T_{U}^{b}(\bar{u}(t)), v(t) \in T_{U}^{b(2)}(\bar{u}(t), u(t))$ for almost all $t \in[0,1]$ and $y(\cdot)$ is the solution of 1.8. In the above $T_{U}^{b}(\bar{u}(t))$ and $T_{U}^{b(2)}(\bar{u}(t), u(t))$ denote, respectively, the first and the second order adjacent tangent sets to $U$ at $\bar{u}(t)$ and $(\bar{u}(t), u(t))$. This second-order optimality condition has been obtained in [18] for the Bolza problem. Its equivalent version for the Mayer problem is proved in Theorem 2.5 below.

The second-order optimality condition 1.9 easily reduces to 1.7$)$ when $\bar{u}(t)$ takes values in the interior of $U$. Indeed, in this case $T_{U}^{b}(\bar{u}(t))=\mathbb{R}^{m}, \mathcal{H}_{u}[t]=0$ due to the maximum principle and $0 \in T_{U}^{b(2)}(\bar{u}(t), u(t))$ for almost all $t \in[0,1]$.

Even when $\bar{u}(t) \in$ Int $U$, the discontinuity of $\bar{u}(\cdot)$ allows it to jump to the boundary in any neighborhood of $t$ and so results known for the case of an open $U$ can not be used. Assuming that $t$ is a Lebesgue point of $\bar{u}(\cdot)$, we are able to apply the Goh transformation and an additional refined analysis to recover the symmetry of the matrix $\mathcal{H}_{x u}[t] f_{u}[t]$ and the negative semi-definiteness of $R(t)$ without any assumptions on $U$. Since for an integrable $\bar{u}(\cdot)$, its set of Lebesgue points has a full measure, for such optimal control both second order necessary optimality conditions are valid almost everywhere in the set $\left\{t \in\left[t_{1}, t_{2}\right]: \bar{u}(t) \in \operatorname{Int} U\right\}$.

If $U$ enjoys some additional structural properties, then for a.e. $t \in\left[t_{1}, t_{2}\right]$ such that $\bar{u}(t) \in \partial U$, the pointwise second order optimality conditions hold true on a subspace of $\mathbb{R}^{m}$. To be more precise, for every $u \in U$ let us consider the maximal subspace $P_{u}$ contained in $T_{U}^{b}(u)$. Note that $P_{u}=\mathbb{R}^{m}$ whenever $u \in \operatorname{Int} U$. In general $P_{\bar{u}(s)}$ is not regular enough with respect to $s$ for two reasons: the lack of regularity of $P_{u}$ with respect to $u$ and the lack of regularity of $\bar{u}(\cdot)$. Still we were able to investigate two important classes of control constraints: the one when $U$ is a convex polytope (typical in the geometric control theory) and the one when the boundary of $U$ is of class $C^{2}$, by considering only variations $u(\cdot)$ taking values in a subspace of $P_{\bar{u}(s)}$ for all $s$.

Below Theorem 4.3 provides pointwise second order necessary optimality conditions in the case when $U$ is a convex polytope and the singular optimal control $\bar{u}(\cdot)$ is merely integrable while Theorem 5.7 deals with $U$ having a $C^{2}$-boundary and an optimal control which is singular and Lipschitz in a neighborhood of some $t \in[0,1]$. In both cases, the symmetry of $\mathcal{H}_{x u}[t] f_{u}[t]$ is 
proved when it is considered as a bilinear operator on the subspace $P_{\bar{u}(t)}$. The negative semidefiniteness of $R(t)$ on $P_{\bar{u}(t)}$ holds true when $U$ is a convex polytope, while when $U$ is of class $C^{2}$ a similar result is valid with a slightly different definition of $R(\cdot)$.

Let us underline that in the case of convex polytopes, we assume $\mathcal{H}_{u u}(\cdot)=0$ on some interval $\left[t_{1}, t_{2}\right]$ to get our results. In the case when the boundary of $U$ is not flat, we assume that for a subspace $S(t) \subset P_{\bar{u}(t)}$ introduced in Section $5,\left(\mathcal{H}_{u u}[t]-\left\|\mathcal{H}_{u}[t]\right\| C(t)\right)_{\mid S(t)}=0$ a.e. in some time interval $\left[t_{1}, t_{2}\right]$, where $C(\cdot)$ is a matrix valued mapping related to the curvature of the boundary of $U$ and defined in Section 5.

As a corollary of our results, in the affine case we obtain the following second order necessary optimality condition for an integrable optimal control $\bar{u}(\cdot)$. Suppose that

$$
f(x, u)=f_{0}(x)+\sum_{l=1}^{m} u_{l} f_{l}(x), \quad u=\left(u_{1}, \ldots, u_{m}\right), \quad u_{l} \in U_{l},
$$

where $f_{l}(\cdot) \in C^{2}\left(\mathbb{R}^{n} ; \mathbb{R}^{n}\right)$ and $U_{l} \subset \mathbb{R}$ is a closed (possibly unbounded) interval for all $l \in$ $\{1, \ldots, m\}$. Consider the matrix valued mapping $\Theta:[0,1] \rightarrow M(m \times m)$, whose elements are

$$
\theta_{k, l}(t):=\left\langle p(t),\left[f_{k}, f_{l}\right](\bar{x}(t))\right\rangle \quad \forall k, l \in\{1, \ldots, m\}
$$

where $\bar{x}(\cdot)$ is the optimal trajectory corresponding to $\bar{u}(\cdot)$ and $p(\cdot)$ is as in the maximum principle. In Corollary 4.4 we prove that for a.e. $t \in[0,1]$, the subspace $\Theta(t)\left(P_{\bar{u}(t)}\right)$ is orthogonal to the subspace $P_{\bar{u}(t)}$. In particular we recover the well known Goh condition in the geometric optimal control: $\Theta(t)=0$ for a.e. $t$ such that $\bar{u}(t) \in \operatorname{Int} U$.

For the Mayer problem with the endpoint constraint $K$, we suppose that the oriented distance $b_{K}(\cdot)$ to $K$ is of class $C^{2}$ on a neighborhood of the boundary of $K$ and we assume that the problem is calm at $\bar{x}(\cdot)$. This allows to reduce it to the one without endpoint constraints, since, by the calmness assumption, for some $k>0,(\bar{x}(\cdot), \bar{u}(\cdot))$ is a strong local minimizer of the "penalized" minimization problem

$$
\min \left\{\varphi(x(1))+k d_{K}(x(1)): x(\cdot) \in S_{[0,1]}\left(x_{0}\right)\right\},
$$

where $d_{K}(\cdot)$ denotes the distance function to $K$. This new problem involves a nondifferentiable cost function and so we do not have the necessary condition $(1.9)$. We proceed then in the following way. Let $q(\cdot)$ be the solution of (1.4) such that $q(1)$ is the unit outward normal to $K$ at $\bar{x}(1)$ and define subspaces $\mathcal{T}_{t} \subset P_{\bar{u}(t)}$ by

$$
\mathcal{T}_{t}:=\left\{u \in P_{\bar{u}(t)}:\left\langle q(t), f_{u}[t] u\right\rangle=0\right\} .
$$

We would like to underline that if the maximum principle of the Mayer problem (1.1) is abnormal, then $\mathcal{T}_{t}=P_{\bar{u}(t)}$. Consider the two solutions $q^{1}(\cdot)$ and $q^{2}(\cdot)$ of 1.4 satisfying

$$
q^{1}(1)=-\nabla \varphi(\bar{x}(1)), \quad q^{2}(1)=-\nabla \varphi(\bar{x}(1))-k \nabla b_{K}(\bar{x}(1) .
$$

As it is explained in Section 6, these are "extremal" transversality conditions for the above penalized problem. In general, the mappings $q^{i}(\cdot)$ do not satisfy the maximum principle (1.5).

Define the functions $\mathcal{H}^{i}(t, \cdot, \cdot)=\mathcal{H}\left(t, \cdot, q^{i}(t), \cdot\right)$ for $i=1,2$. In the case of a convex polytope $U$ our second order necessary optimality conditions state that if for some $0 \leq t_{1}<t_{2} \leq 1$ and for $j=1,2$ we have $\mathcal{H}_{u u}^{j}[\cdot]=0$ on $\left[t_{1}, t_{2}\right]$, then for a.e. $t \in\left[t_{1}, t_{2}\right]$ there exists $i \in\{1,2\}$ such that $\mathcal{H}_{x u}^{i}[t] f_{u}[t]$ is symmetric on $\mathcal{T}_{t}$ and

$$
\frac{1}{2} f_{u}[t]^{*} \mathcal{H}_{x x}^{i}[t] f_{u}[t]-\frac{1}{2} \frac{d}{d t} \mathcal{H}_{x u}^{i}[t] f_{u}[t]-\mathcal{H}_{x u}^{i}[t] f_{x}[t] f_{u}[t]+\frac{1}{2} \mathcal{H}_{x u}^{i}[t] \frac{d}{d t} f_{u}[t]
$$

is negative semi-definite on $\mathcal{T}_{t}$. To prove these results, we use the variational approach developed for the Mayer problem without endpoint constraints.

For a detailed survey of the origin of high order necessary conditions for optimality, see Gabasov and Kirillova [14, and the reference therein. There, when dealing with closed control 
constraints, the authors gave second-order optimality conditions for a different definition of singular trajectory/control pair, which reduces to ours in the case of an open set $U$.

In [19] and 20], for the first time, Krener investigated the Goh condition (and its higher order extensions) when endpoint constraints are present and the optimal control is piecewise $C^{\infty}$, by using technics of the geometric control theory. This approach was taken over by several authors and the Goh condition became mostly known in the form involving Lie brackets, as a secondorder necessary optimality condition for the abnormal maximum principle (see Remark 3.2 below). Indeed, the Lie brackets describing second-order variations of the endpoint mapping, some authors studied the Goh condition in the context of such mapping, rather than for the cost function. See [3] for a classical derivation of the Goh condition for essentially bounded abnormal optimal controls and open control constraints. In [22, pp.314-319], the Goh condition is derived for a trajectory ending on the boundary of the reachable set of an affine control system with a corresponding control taking values in the interior of control constraints. In [1], abnormal second order optimality conditions were investigated for an affine control system with the control constraint in the form of a closed convex polytope and endpoint constraints, see also [2. There, second order necessary optimality conditions are expressed using the Lie brackets at points of continuity of the optimal control. In our approach, when $U$ is a convex polytope, we do not need to assume such continuity. Finally, let us mention [4, where for a specific control problem with closed set of controls, the Goh condition was derived for essentially bounded controls.

Several authors made an extensive use of the Goh transformation to recover other necessary and sufficient second order optimality conditions. In [12] and [13, Dmitruk considered optimal control problems which are linear in the control and with closed control constraints. Recently, Aronna et al. [6] et Aronna [5, proved an integral and a pointwise forms of second order necessary and sufficient optimality conditions for bang-singular optimal controls with endpoint constraints, in the affine and partially affine cases. Applying the Goh transformation, they reduced the singular classical second-order necessary optimality condition (1.7) to a new one, which includes a quadratic term in the control variation. This implies a pointwise second order condition. However, they imposed strong structural assumptions on optimal controls, cf. Assumptions 1 and 2 in [6], that are difficult to check.

In conclusion we propose here pointwise conditions similar to those of Goh and the generalized Legendre-Clebsch conditions in the presence of a closed control constraint. This is done by investigating first the free endpoint problem. To attempt the case of endpoint constraints we add the calmness assumption to reduce the Mayer problem with endpoint constraints to one without them, but involving a nondifferentiable cost function. For this equivalent problem we obtain similar pointwise conditions using the developed variational approach for the free endpoint problems.

The paper is organized as follows. In Section 2 we introduce some notations and provide some preliminary results. In Section 3 we derive the second order necessary optimality conditions for an arbitrary set $U$ and any measurable optimal control at times when it belongs to the interior of $U$. Section 4 deals with the case when $U$ is a convex polytope and Section 5 with the case when $U$ is of class $C^{2}$. The last Section is devoted to the second order necessary optimality conditions for the Mayer problem involving endpoint constraints.

\section{Preliminaries}

All along the paper the norm in $\mathbb{R}^{n}$ is denoted by $\|\cdot\|$ and the inner product by $\langle\cdot, \cdot\rangle$. Let $B:=\left\{x \in \mathbb{R}^{n}:\|x\| \leq 1\right\}$ be the closed unit ball and $\stackrel{\circ}{B}$ its interior.

Definition 2.1. Let $C: \mathbb{R}^{m} \rightarrow \mathbb{R}^{m}$ be a linear mapping and $F$ be a subspace of $\mathbb{R}^{m}$. We say that $C$ is symmetric on $F$ if $\langle C u, v\rangle=\langle u, C v\rangle$ for all $u, v \in F$ and that $C$ is negative semi-definite on $F$ if $\langle C u, u\rangle \leq 0$ for all $u \in F$. 
Notice that $C$ is symmetric on $F$ if and only if $C-C^{*}$ maps $F$ into its orthogonal space $F^{\perp}$, where $C^{*}$ is the transpose of $C$.

For a nonempty subset $K$ of $\mathbb{R}^{n}, \partial K$ denotes its boundary, Int $K$ its interior and $K^{c}$ its complement. The distance function $d_{K}: \mathbb{R}^{n} \rightarrow \mathbb{R}_{+}$is defined by $d_{K}(x):=\inf _{y \in K}\|x-y\|$ for all $x \in \mathbb{R}^{n}$ and the oriented distance $b_{K}: \mathbb{R}^{n} \rightarrow \mathbb{R}$ for $K \neq \mathbb{R}^{n}$ by $b_{K}(x):=d_{K}(x)-d_{K^{c}}(x)$ for all $x \in \mathbb{R}^{n}$. We set $b_{K}(\cdot)=0$ if $K=\mathbb{R}^{n}$.

Let $U$ be a nonempty closed subset of $\mathbb{R}^{m}$. For $u_{0} \in U$ the adjacent tangent cone to $U$ at $u_{0}$ is defined by

$$
T_{U}^{b}\left(u_{0}\right):=\left\{u \in \mathbb{R}^{n}: \lim _{h \rightarrow 0+} \frac{d_{U}\left(u_{0}+h u\right)}{h}=0\right\} .
$$

Further for $u \in \mathbb{R}^{n}$, the second-order adjacent subset to $U$ at $\left(u_{0}, u\right)$ is the set defined by

$$
T_{U}^{b(2)}\left(u_{0}, u\right):=\left\{v \in \mathbb{R}^{n}: \lim _{h \rightarrow 0+} \frac{d_{U}\left(u_{0}+h u+h^{2} v\right)}{h^{2}}=0\right\} .
$$

In this paper for any $u \in U$ we denote by

$$
P_{u} \text { the largest subspace contained in } T_{U}^{b}(u) \text {. }
$$

Consider $f:[0,1] \times \mathbb{R}^{n} \times \mathbb{R}^{m} \rightarrow \mathbb{R}^{n}$. Below $f^{\prime}(t, x, u)$ denotes the derivative of the map $(x, u) \mapsto f(t, x, u)$, while $f_{x}(t, x, u)$ and $f_{u}(t, x, u)$ the partial derivatives of $f$ with respect to $x$ and $u ; f^{\prime \prime}(t, x, u)$ denotes the Hessian of the map $(x, u) \mapsto f(t, x, u), f_{x x}(t, x, u), f_{x u}(t, x, u)$ and $f_{u u}(t, x, u)$ the second-order partial derivative of $f$ with respect to $x$ and $u$.

Fix a trajectory/control pair $(\bar{x}(\cdot), \bar{u}(\cdot))$ of 11.2 and set $f[t]=f(t, \bar{x}(t), \bar{u}(t))$. Notations $f_{u}[t], f_{x}[t]$ and $f^{\prime \prime}[t]$ are defined in a similar way.

Throughout the whole paper we impose the following assumptions : For some $\delta>0$

(a) $\left\|f_{u}[\cdot]\right\| \in L^{1}\left([0,1] ; \mathbb{R}_{+}\right)$and $\exists a_{1}(\cdot) \in L^{1}\left([0,1] ; \mathbb{R}_{+}\right)$such that for a.e. $t \in[0,1]$

$$
\begin{aligned}
& \left\|f_{u}(t, x, u)-f_{u}(t, y, v)\right\| \leq a_{1}(t)(\|x-y\|+\|u-v\|), \\
& \forall x, y \in \bar{x}(t)+\delta \stackrel{\circ}{B}, \forall u, v \in \bar{u}(t)+\delta \stackrel{\circ}{B}
\end{aligned}
$$

(b) For a.e. $t \in[0,1], \forall x \in \bar{x}(t)+\delta B, f(t, x, U)$ is a closed set;

(c) For all $t \in[0,1], f(t, \cdot, \cdot)$ is twice differentiable on $(\bar{x}(t)+\delta \stackrel{\circ}{B}) \times(\bar{u}(t)+\delta \stackrel{\circ}{B})$;

(d) $\left\|f^{\prime}[\cdot]\right\|+\left\|f^{\prime \prime}[\cdot]\right\| \in L^{1}\left([0,1] ; \mathbb{R}_{+}\right)$and $\exists a_{2}(\cdot) \in L^{1}\left([0,1] ; \mathbb{R}_{+}\right)$such that for a.e. $t \in[0,1]$ and all $x, y \in \bar{x}(t)+\delta \dot{B}, u, v \in \bar{u}(t)+\delta \dot{B}$

$$
\left\|f^{\prime}(t, x, u)-f^{\prime}(t, y, v)\right\|+\left\|f^{\prime \prime}(t, x, u)-f^{\prime \prime}(t, y, v)\right\| \leq a_{2}(t)(\|x-y\|+\|u-v\|) ;
$$

(e) $\varphi(\cdot) \in C^{2}$ on $\bar{x}(1)+\delta \stackrel{\circ}{B}$.

The above hypotheses are sufficient to ensure the validity of the maximum principle and of 1.9 ).

Definition 2.2. A trajectory/control pair $(\bar{x}(\cdot), \bar{u}(\cdot))$ is a strong local minimizer for 1.3) (resp. (1.1)) if there exists $\eta>0$ such that $\bar{x}(\cdot)$ minimizes $\varphi$ over all $x(\cdot) \in S_{[0,1]}\left(x_{0}\right)$ (resp. over all $x(\cdot) \in S_{[0,1]}\left(x_{0}\right)$ with $\left.x(1) \in K\right)$ satisfying $\|\bar{x}(\cdot)-x(\cdot)\|_{\infty} \leq \eta$.

Theorem 2.3 (Maximum Principle). Let $(\bar{x}(\cdot), \bar{u}(\cdot))$ be a strong local minimizer for the Mayer problem (1.3). Then there exists an absolutely continuous $p:[0,1] \rightarrow \mathbb{R}^{n}$ satisfying (1.4), (1.5) and (1.6).

Lemma 2.4. Let $(\bar{x}(\cdot), \bar{u}(\cdot))$ be a strong local minimizer of (1.3) and $p(\cdot)$ be as in the maximum principle of Theorem 2.3. Then for a.e. $t \in[0,1]$ and for any $u \in P_{\bar{u}(t)}$ we have $\mathcal{H}_{u}[t] u=0$.

Proof. Fix $t \in[0,1]$ for which $[1.5]$ is verified and let $u \in P_{\bar{u}(t)}$. Then for every $h>0$ there exists $u_{h}$ such that $\bar{u}(t)+h u_{h} \in U$ and $\lim _{h \rightarrow 0+} u_{h}=u$. Hence $\mathcal{H}\left(t, \bar{x}(t), p(t), \bar{u}(t)+h u_{h}\right)-$ $\mathcal{H}(t, \bar{x}(t), p(t), \bar{u}(t)) \leq 0$. Dividing by $h>0$ and letting $h \rightarrow 0+$, we obtain $\mathcal{H}_{u}[t] u \leq 0$. Since $-u$ belongs to $P_{\bar{u}(t)}$, the same inequality holds for $-u$, implying that $\mathcal{H}_{u}[t] u=0$. 
We introduce the following two systems, which will play an important role throughout the paper

$$
\begin{gathered}
\left\{\begin{array}{l}
y^{\prime}(t)=f_{x}[t] y(t)+f_{u}[t] u(t) \\
y(0)=0,
\end{array}\right. \\
\left\{\begin{array}{l}
w^{\prime}(t)=f_{x}[t] w(t)+f_{u}[t] v(t)+\frac{1}{2} y(t)^{*} f_{x x}[t] y(t)+u(t)^{*} f_{x u}[t] y(t)+\frac{1}{2} u(t)^{*} f_{u u}[t] u(t) \\
w(0)=0 .
\end{array}\right.
\end{gathered}
$$

Define $A:=\left\{t \in[0,1]: \bar{u}(t) \in \partial U, \mathcal{H}_{u}[t] \neq 0\right\}$ and

$\mathcal{U}_{A}=\left\{u(\cdot) \in L^{\infty}\left([0,1] ; \mathbb{R}^{m}\right): \exists c>0, h_{0}>0\right.$ s.t. $\left.\forall h \in\left[0, h_{0}\right], \forall t \in A, d_{U}(\bar{u}(t)+h u(t)) \leq c h^{2}\right\}$.

Note that any $u(\cdot) \in \mathcal{U}_{A}$ satisfies $u(t) \in T_{U}^{b}(\bar{u}(t))$ for all $t \in A$.

Theorem 2.5. Let $(\bar{x}(\cdot), \bar{u}(\cdot))$ be a strong local minimizer for the Mayer problem (1.3) and $p(\cdot)$ be as in the maximum principle of Theorem 2.3. Then for all $u(\cdot), v(\cdot) \in L^{\infty}\left([0,1] ; \mathbb{R}^{m}\right)$ such that $u(\cdot) \in \mathcal{U}_{A}$ and $v(t) \in T_{U}^{b(2)}(\bar{u}(t), u(t))$ for a.e. $t \in A$, either $\mathcal{H}_{u}[t] u(t)<0$ on a set of positive measure or $\mathcal{H}_{u}[t] u(t)=0$ a.e. in $[0,1]$ and (1.9) holds true, where $y(\cdot)$ is the solution of (2.1).

Proof. Let $u(\cdot), v(\cdot)$ be as in the statement of the theorem. The inequality $\mathcal{H}_{u}[t] u(t) \leq 0$ for a.e. $t$ in $[0,1]$ follows from the maximum principle. Thus it remains to prove $(1.9)$ when $\mathcal{H}_{u}[t] u(t)=0$ a.e. Consider the solution $y(\cdot)$ of (2.1) and the solution $w(\cdot)$ of $(2.2)$. By Propositions 4.2 and 4.4 from [18, for every $h>0$ there exists $v_{h}(\cdot) \in L^{\infty}\left([0,1], \mathbb{R}^{m}\right)$ such that $v_{h}(\cdot)$ converge a.e. to $v(\cdot)$ when $h \rightarrow 0+,\left\{\left\|v_{h}\right\|_{\infty}\right\}_{h>0}$ is bounded, and for $\tilde{u}_{h}(\cdot)=\bar{u}(\cdot)+h u(\cdot)+h^{2} v_{h}(\cdot)$, and for every $h>0$ small enough, the solution $\tilde{x}_{h}(\cdot)$ to 1.2 with $u(\cdot)=\tilde{u}_{h}(\cdot)$ is well defined and $\frac{\tilde{x}_{h}-\bar{x}-h y}{h^{2}}$ converge uniformly to $w(\cdot)$ when $h \rightarrow 0+$. Thus for our minimization problem we have

$$
0 \geq \varphi(\bar{x}(1))-\varphi\left(\tilde{x}_{h}(1)\right)=-\left\langle\nabla \varphi(\bar{x}(1)), h y(1)+h^{2} w(1)\right\rangle-\frac{h^{2}}{2} y(1)^{*} \varphi^{\prime \prime}(\bar{x}(1)) y(1)+o\left(h^{2}\right) .
$$

On the other hand, thanks to the transversality condition $(1.6)$ it follows

$$
\begin{aligned}
- & \langle\nabla \varphi(\bar{x}(1)), y(1)\rangle=\langle p(1), y(1)\rangle-\langle p(0), y(0)\rangle=\int_{0}^{1}\langle p, y\rangle^{\prime}(t) d t \\
& =-\int_{0}^{1} \mathcal{H}_{x}[t] y(t) d t+\int_{0}^{1}\left(\left\langle p(t), f_{x}[t] y(t)+f_{u}[t] u(t)\right\rangle\right) d t=\int_{0}^{1} \mathcal{H}_{u}[t] u(t) d t=0 .
\end{aligned}
$$

Therefore, $\langle\nabla \varphi(\bar{x}(1)), y(1)\rangle=0$. Hence, dividing $(2.3)$ by $h^{2}$ and letting $h \rightarrow 0$ yields

$$
\left.0 \geq-\langle\nabla \varphi(\bar{x}(1)), w(1)\rangle-\frac{1}{2} y(1)\right)^{*} \varphi^{\prime \prime}(\bar{x}(1)) y(1) .
$$

Using again the transversality condition we obtain

$$
\begin{aligned}
-\langle\nabla \varphi(\bar{x}(1)), w(1)\rangle & =\langle p(1), w(1)\rangle-\langle p(0), w(0)\rangle=\int_{0}^{1}\langle p, w\rangle^{\prime}(t) d t \\
& =-\int_{0}^{1} \mathcal{H}_{x}[t] w(t) d t+\int_{0}^{1}\left\langle p(t), w^{\prime}(t)\right\rangle d t
\end{aligned}
$$

Therefore, by (2.2), inequality (2.4) implies (1.9).

For a Lebesgue measurable set $E \subset[0,1]$, let $|E|$ denote its Lebesgue measure. To say that a property holds true a.e. in $E$ means that for a subset $D \subset E$ having $|D|=0$ this property is verified for all $t \in E \backslash D$. When $|E|=0$, then $E \backslash D$ may be an empty set. 
Let $a:[0,1] \rightarrow \mathbb{R}^{k}$ be an integrable function. We recall that the set of its Lebesgue points has full measure in $[0,1]$. If $t \in(0,1)$ is a Lebesgue point, then for every $\varepsilon>0$ there exists $\delta_{\varepsilon}>0$ such that

$$
\lim _{\varepsilon \rightarrow 0+} \delta_{\varepsilon}=0 \quad \text { and } \quad \frac{1}{2 \delta_{\varepsilon}} \int_{t-\delta_{\varepsilon}}^{t+\delta_{\varepsilon}}\|a(s)-a(t)\| d s \leq \varepsilon^{2} .
$$

Thus

$$
\left|\left\{s \in\left(t-\delta_{\varepsilon}, t+\delta_{\varepsilon}\right) \mid\|a(s)-a(t)\| \geq \varepsilon\right\}\right| \leq 2 \varepsilon \delta_{\varepsilon} .
$$

For every $\varepsilon>0$ define the measurable set (depending on $t$ )

$$
E_{\varepsilon}:=\left\{s \in\left(t-\delta_{\varepsilon}, t+\delta_{\varepsilon}\right): s \text { is a Lebesgue point of } a(\cdot) \text { and }\|a(s)-a(t)\|<\varepsilon\right\} .
$$

Consider next a Lebesgue measurable $\Upsilon \subset(0,1)$ and define the function $\phi_{\varepsilon}(s):=\int_{0}^{s} \chi_{\Upsilon \cap E_{\varepsilon}}(\tau) d \tau$, where $\chi \Upsilon \cap E_{\varepsilon}$ denotes the characteristic function of $\Upsilon \cap E_{\varepsilon}$. Then $\phi_{\varepsilon}(s)=0$ for all $s \in\left[0, t-\delta_{\varepsilon}\right]$, $\phi_{\varepsilon}\left(t+\delta_{\varepsilon}\right)=\left|\Upsilon \cap E_{\varepsilon}\right|$ and, by the intermediate value theorem, there exists $t_{\varepsilon} \in\left(t-\delta_{\varepsilon}, t+\delta_{\varepsilon}\right)$ such that $\phi_{\varepsilon}\left(t+\delta_{\varepsilon}\right)=2 \phi_{\varepsilon}\left(t_{\varepsilon}\right)$. Set

$$
E_{\varepsilon}^{1}:=\Upsilon \cap E_{\varepsilon} \cap\left[0, t_{\varepsilon}\right], \quad E_{\varepsilon}^{2}:=\Upsilon \cap E_{\varepsilon} \cap\left[t_{\varepsilon}, 1\right] .
$$

Then $\left|E_{\varepsilon}^{1}\right|=\left|E_{\varepsilon}^{2}\right|=\frac{1}{2}\left|\Upsilon \cap E_{\varepsilon}\right|$. We will need the following lemma.

Lemma 2.6. Let $a(\cdot):[0,1] \rightarrow \mathbb{R}^{k}$ be an integrable function, $\Upsilon \subset(0,1)$ be a measurable set with $|\Upsilon|>0, t \in(0,1) \cap \Upsilon$ be a Lebesgue point of $a(\cdot)$ whose Lebesgue density in $\Upsilon$ is equal to one. Then for $\delta_{\varepsilon}, E_{\varepsilon}, E_{\varepsilon}^{1}, E_{\varepsilon}^{2}$ defined as above we have

$$
\lim _{\varepsilon \rightarrow 0+} \frac{\left|\Upsilon \cap E_{\varepsilon}\right|}{2 \delta_{\varepsilon}}=1
$$

and for all small $\varepsilon>0$

$$
\begin{aligned}
& \frac{1}{\left|E_{\varepsilon}^{1}\right|^{2}} \int_{t-\delta_{\varepsilon}}^{t+\delta_{\varepsilon}}\left(\left|E_{\varepsilon}^{1} \cap[0, s]\right|-\left|E_{\varepsilon}^{2} \cap[0, s]\right|\right)^{2} d s=\frac{1}{3}\left|\Upsilon \cap E_{\varepsilon}\right|+o\left(\left|\Upsilon \cap E_{\varepsilon}\right|\right), \\
& \frac{1}{\left|E_{\varepsilon}^{1}\right|} \int_{t-\delta_{\varepsilon}}^{t+\delta_{\varepsilon}}\left(\left|E_{\varepsilon}^{1} \cap[0, s]\right|-\left|E_{\varepsilon}^{2} \cap[0, s]\right|\right) \chi \Upsilon \cap E_{\varepsilon}(s) d s=\frac{1}{2}\left|\Upsilon \cap E_{\varepsilon}\right|+o\left(\left|\Upsilon \cap E_{\varepsilon}\right|\right),
\end{aligned}
$$

where the notation $o\left(\left|\Upsilon \cap E_{\varepsilon}\right|\right)$ means $\lim _{\varepsilon \rightarrow 0+} \frac{o\left(\left|\Upsilon \cap E_{\varepsilon}\right|\right)}{\left|\Upsilon \cap E_{\varepsilon}\right|}=0$.

Proof. Since $t$ has the Lebesgue density equal to one in $\Upsilon$, we have

$$
\lim _{\varepsilon \rightarrow 0+} \frac{\left|\Upsilon \cap\left(t-\delta_{\varepsilon}, t+\delta_{\varepsilon}\right)\right|}{2 \delta_{\varepsilon}}=1
$$

By (2.6), $\left|\left(\Upsilon \cap\left(t-\delta_{\varepsilon}, t+\delta_{\varepsilon}\right)\right) \backslash E_{\varepsilon}\right| \leq\left|\left(t-\delta_{\varepsilon}, t+\delta_{\varepsilon}\right) \backslash E_{\varepsilon}\right| \leq 2 \varepsilon \delta_{\varepsilon}$ and from the equality

$$
\left|\Upsilon \cap\left(t-\delta_{\varepsilon}, t+\delta_{\varepsilon}\right)\right|=\left|\Upsilon \cap E_{\varepsilon}\right|+\left|\left(\Upsilon \cap\left(t-\delta_{\varepsilon}, t+\delta_{\varepsilon}\right)\right) \backslash E_{\varepsilon}\right|
$$

we deduce $(2.9)$. Observe that $(2.12)$ and $(2.9)$ yield

$$
\lim _{\varepsilon \rightarrow 0+} \frac{\left|\Upsilon \cap\left(t-\delta_{\varepsilon}, t+\delta_{\varepsilon}\right)\right|}{\left|\Upsilon \cap E_{\varepsilon}\right|}=1 .
$$

Therefore, from (2.9) and the equality $\left|\left(t-\delta_{\varepsilon}, t+\delta_{\varepsilon}\right)\right|=\left|\Upsilon \cap E_{\varepsilon}\right|+\left|\left(t-\delta_{\varepsilon}, t+\delta_{\varepsilon}\right) \backslash\left(\Upsilon \cap E_{\varepsilon}\right)\right|$ we obtain

$$
\lim _{\varepsilon \rightarrow 0+} \frac{\left|\left(t-\delta_{\varepsilon}, t+\delta_{\varepsilon}\right) \backslash\left(\Upsilon \cap E_{\varepsilon}\right)\right|}{\left|\Upsilon \cap E_{\varepsilon}\right|}=0 .
$$

The inclusions

$$
\left(t-\delta_{\varepsilon}, t_{\varepsilon}\right) \backslash E_{\varepsilon}^{1} \subset\left(t-\delta_{\varepsilon}, t+\delta_{\varepsilon}\right) \backslash\left(\Upsilon \cap E_{\varepsilon}\right) \&\left(t_{\varepsilon}, t+\delta_{\varepsilon}\right) \backslash E_{\varepsilon}^{2} \subset\left(t-\delta_{\varepsilon}, t+\delta_{\varepsilon}\right) \backslash\left(\Upsilon \cap E_{\varepsilon}\right)
$$


and 2.13) imply that

$$
\lim _{\varepsilon \rightarrow 0+} \frac{\left|\left(t-\delta_{\varepsilon}, t_{\varepsilon}\right) \backslash E_{\varepsilon}^{1}\right|}{\left|\Upsilon \cap E_{\varepsilon}\right|}=\lim _{\varepsilon \rightarrow 0+} \frac{\left|\left(t_{\varepsilon}, t+\delta_{\varepsilon}\right) \backslash E_{\varepsilon}^{2}\right|}{\left|\Upsilon \cap E_{\varepsilon}\right|}=0 .
$$

Notice that

$$
\begin{aligned}
\int_{t-\delta_{\varepsilon}}^{t+\delta_{\varepsilon}}\left(\left|E_{\varepsilon}^{1} \cap[0, s]\right|\right. & \left.-\left|E_{\varepsilon}^{2} \cap[0, s]\right|\right)^{2} d s= \\
& =\int_{t-\delta_{\varepsilon}}^{t_{\varepsilon}}\left(\int_{t-\delta_{\varepsilon}}^{s} \chi_{E_{\varepsilon}^{1}}(\tau) d \tau\right)^{2} d s+\int_{t_{\varepsilon}}^{t+\delta_{\varepsilon}}\left(\left|E_{\varepsilon}^{1}\right|-\int_{t_{\varepsilon}}^{s} \chi_{E_{\varepsilon}^{2}}(\tau) d \tau\right)^{2} d s .
\end{aligned}
$$

For the first term we have

$$
\begin{aligned}
& \int_{t-\delta_{\varepsilon}}^{t_{\varepsilon}}\left(\int_{t-\delta_{\varepsilon}}^{s} \chi_{E_{\varepsilon}^{1}}(\tau) d \tau\right)^{2} d s \\
& \quad=\int_{t-\delta_{\varepsilon}}^{t_{\varepsilon}} \chi_{E_{\varepsilon}^{1}}(s)\left(\int_{t-\delta_{\varepsilon}}^{s} \chi_{E_{\varepsilon}^{1}}(\tau) d \tau\right)^{2} d s+\int_{t-\delta_{\varepsilon}}^{t_{\varepsilon}} \chi_{\left[t-\delta_{\varepsilon}, t_{\varepsilon}\right] \backslash E_{\varepsilon}^{1}}(s)\left(\int_{t-\delta_{\varepsilon}}^{s} \chi_{E_{\varepsilon}^{1}}(\tau) d \tau\right)^{2} d s \\
& \quad=\int_{t-\delta_{\varepsilon}}^{t_{\varepsilon}} \frac{1}{3}\left(\left(\int_{t-\delta_{\varepsilon}}^{s} \chi_{E_{\varepsilon}^{1}}(\tau) d \tau\right)^{3}\right)^{\prime} d s+a_{\varepsilon}=\frac{1}{3}\left|E_{\varepsilon}^{1}\right|^{3}+a_{\varepsilon},
\end{aligned}
$$

where

$$
a_{\varepsilon}:=\int_{t-\delta_{\varepsilon}}^{t_{\varepsilon}} \chi_{\left[t-\delta_{\varepsilon}, t_{\varepsilon}\right] \backslash E_{\varepsilon}^{1}}(s)\left(\int_{t-\delta_{\varepsilon}}^{s} \chi_{E_{\varepsilon}^{1}}(\tau) d \tau\right)^{2} d s
$$

and, thanks to 2.14), $a_{\varepsilon} \leq\left|\left(t-\delta_{\varepsilon}, t_{\varepsilon}\right) \backslash E_{\varepsilon}^{1}\right|\left|E_{\varepsilon}^{1}\right|^{2}=o\left(\left|\Upsilon \cap E_{\varepsilon}\right|\right)\left|E_{\varepsilon}^{1}\right|^{2}$.

Since $\left|E_{\varepsilon}^{1}\right|=\left|E_{\varepsilon}^{2}\right|$, for the second term we have

$$
\begin{aligned}
\int_{t_{\varepsilon}}^{t+\delta_{\varepsilon}} & \left(\left|E_{\varepsilon}^{2}\right|-\int_{t_{\varepsilon}}^{s} \chi_{E_{\varepsilon}^{2}}(\tau) d \tau\right)^{2} d s=\int_{t_{\varepsilon}}^{t+\delta_{\varepsilon}}\left|E_{\varepsilon}^{2} \cap\left[s, t+\delta_{\varepsilon}\right]\right|^{2} d s=\int_{t_{\varepsilon}}^{t+\delta_{\varepsilon}}\left(\int_{s}^{t+\delta_{\varepsilon}} \chi_{E_{\varepsilon}^{2}}(\tau) d \tau\right)^{2} d s \\
= & \int_{t_{\varepsilon}}^{t+\delta_{\varepsilon}} \chi_{E_{\varepsilon}^{2}}(s)\left(\int_{s}^{t+\delta_{\varepsilon}} \chi_{E_{\varepsilon}^{2}}(\tau) d \tau\right)^{2} d s+\int_{t_{\varepsilon}}^{t+\delta_{\varepsilon}} \chi_{\left[t_{\varepsilon}, t+\delta_{\varepsilon}\right] \backslash E_{\varepsilon}^{2}}(s)\left(\int_{s}^{t+\delta_{\varepsilon}} \chi_{E_{\varepsilon}^{2}}(\tau) d \tau\right)^{2} d s \\
= & -\frac{1}{3} \int_{t_{\varepsilon}}^{t+\delta_{\varepsilon}}\left(\left(\int_{s}^{t+\delta_{\varepsilon}} \chi_{E_{\varepsilon}^{2}}(\tau) d \tau\right)^{3}\right)^{\prime} d s+b_{\varepsilon}=\frac{1}{3}\left|E_{\varepsilon}^{2}\right|^{3}+b_{\varepsilon},
\end{aligned}
$$

where

$$
b_{\varepsilon}:=\int_{t_{\varepsilon}}^{t+\delta_{\varepsilon}} \chi_{\left[t_{\varepsilon}, t+\delta_{\varepsilon}\right] \backslash E_{\varepsilon}^{2}}(s)\left(\int_{s}^{t+\delta_{\varepsilon}} \chi_{E_{\varepsilon}^{2}}(\tau) d \tau\right)^{2} d s
$$

and, thanks to (2.14), $b_{\varepsilon} \leq\left|\left(t_{\varepsilon}, t+\delta_{\varepsilon}\right) \backslash E_{\varepsilon}^{2}\right|\left|E_{\varepsilon}^{2}\right|^{2}=o\left(\left|\Upsilon \cap E_{\varepsilon}\right|\right)\left|E_{\varepsilon}^{2}\right|^{2}$.

Dividing by $\left|E_{\varepsilon}^{1}\right|^{2}$ and recalling that $\left|E_{\varepsilon}^{1}\right|=\left|E_{\varepsilon}^{2}\right|=\frac{1}{2}\left|\Upsilon \cap E_{\varepsilon}\right|$, we deduce 2.10].

Using similar arguments it can be shown that

$$
\frac{1}{\left|E_{\varepsilon}^{1}\right|} \int_{t-\delta_{\varepsilon}}^{t+\delta_{\varepsilon}}\left(\left|E_{\varepsilon}^{1} \cap[0, s]\right|-\left|E_{\varepsilon}^{2} \cap[0, s]\right|\right) d s=\frac{1}{2}\left|\Upsilon \cap E_{\varepsilon}\right|+o\left(\left|\Upsilon \cap E_{\varepsilon}\right|\right) .
$$

On the other hand

$$
\begin{aligned}
\int_{t-\delta_{\varepsilon}}^{t+\delta_{\varepsilon}} & \left(\left|E_{\varepsilon}^{1} \cap[0, s]\right|-\left|E_{\varepsilon}^{2} \cap[0, s]\right|\right) d s=\int_{t-\delta_{\varepsilon}}^{t+\delta_{\varepsilon}}\left(\left|E_{\varepsilon}^{1} \cap[0, s]\right|-\left|E_{\varepsilon}^{2} \cap[0, s]\right|\right) \chi_{\Upsilon \cap E_{\varepsilon}}(s) d s \\
& +\int_{t-\delta_{\varepsilon}}^{t+\delta_{\varepsilon}}\left(\left|E_{\varepsilon}^{1} \cap[0, s]\right|-\left|E_{\varepsilon}^{2} \cap[0, s]\right|\right) \chi_{\left(t-\delta_{\varepsilon}, t+\delta_{\varepsilon}\right) \backslash\left(\Upsilon \cap E_{\varepsilon}\right)}(s) d s .
\end{aligned}
$$

Since $0 \leq\left|E_{\varepsilon}^{1} \cap[0, s]\right|-\left|E_{\varepsilon}^{2} \cap[0, s]\right| \leq\left|E_{\varepsilon}^{1}\right|$ for every $s \in\left(t-\delta_{\varepsilon}, t+\delta_{\varepsilon}\right)$ and $\left|\left(t-\delta_{\varepsilon}, t+\delta_{\varepsilon}\right) \backslash\left(\Upsilon \cap E_{\varepsilon}\right)\right|=$ $o\left(\left|\Upsilon \cap E_{\varepsilon}\right|\right)$ we obtain (2.11). 
Let $\mathcal{R}:[0,1] \rightarrow M(l \times l), l \in \mathbb{N}$ be a matrix-valued function. In this paper we denote by $m^{*}(t)$ the largest integer in $\{1, \ldots, l\}$ such that for some matrices $\mathcal{R}_{0}(t) \in M\left(m^{*}(t) \times m^{*}(t)\right), \mathcal{R}_{1}(t) \in$ $M\left(m^{*}(t) \times\left(l-m^{*}(t)\right)\right), \mathcal{R}_{2}(t) \in M\left(\left(l-m^{*}(t)\right) \times m^{*}(t)\right), \mathcal{R}_{3}(t) \in M\left(\left(l-m^{*}(t)\right) \times\left(l-m^{*}(t)\right)\right)$ we have

$$
\mathcal{R}_{0}(t)=\mathcal{R}_{0}(t)^{*}, \quad \mathcal{R}(t)=\left[\begin{array}{ll}
\mathcal{R}_{0}(t) & \mathcal{R}_{1}(t) \\
\mathcal{R}_{2}(t) & \mathcal{R}_{3}(t)
\end{array}\right] .
$$

If $m^{*}(t)=l$, then $\mathcal{R}_{1}(t), \mathcal{R}_{2}(t), \mathcal{R}_{3}(t)$ are absent in the above partition.

\section{SECOND ORDER CONDITIONS IN THE INTERIOR OF CONTROL CONSTRAINTS}

Let $(\bar{x}(\cdot), \bar{u}(\cdot))$ be a strong local minimizer for the Mayer problem 1.3$)$ and $p(\cdot)$ be as in the maximum principle of Theorem 2.3 .

In this section we analyze the points $t \in[0,1]$ such that $\bar{u}(t) \in \operatorname{Int} U$.

Theorem 3.1. Assume that for some $0 \leq t_{1}<t_{2} \leq 1, \bar{u}(\cdot)$ is integrable on $\left[t_{1}, t_{2}\right]$ and

$$
\left\{\begin{aligned}
(i) & \mathcal{H}_{u u}[t]=0 \text { a.e. in }\left[t_{1}, t_{2}\right] ; \\
(\text { ii }) & \mathcal{H}_{x u}[\cdot], f_{u}[\cdot] \text { are Lipschitz on }\left[t_{1}, t_{2}\right] ; \\
(\text { iii }) & \mathcal{H}_{x x}[\cdot], f_{x}[\cdot] \text { are essentially bounded on }\left[t_{1}, t_{2}\right] .
\end{aligned}\right.
$$

Then, for a.e. $t \in\left[t_{1}, t_{2}\right]$ such that $\bar{u}(t) \in \operatorname{Int} U$, the matrix $\mathcal{H}_{x u}[t] f_{u}[t]$ is symmetric and

$$
R(t):=\frac{1}{2} f_{u}[t]^{*} \mathcal{H}_{x x}[t] f_{u}[t]-\frac{1}{2} \frac{d}{d t} \mathcal{H}_{x u}[t] f_{u}[t]-\mathcal{H}_{x u}[t] f_{x}[t] f_{u}[t]+\frac{1}{2} \mathcal{H}_{x u}[t] \frac{d}{d t} f_{u}[t] \leq 0 .
$$

Consequently, if $t \in\left[t_{1}, t_{2}\right]$ is such that for some $\delta>0, u(s)$ is in the interior of $U$ for a.e. $s \in[t-\delta, t+\delta] \cap\left[t_{1}, t_{2}\right]$, then $\mathcal{H}_{x u}[t] f_{u}[t]$ is symmetric.

Remark 3.2. In the case of $f$ affine with respect to controls, the symmetry of the matrix $\mathcal{H}_{x u}[\cdot] f_{u}[\cdot]$ implies that the adjoint state is orthogonal to the Lie brackets of the flux. Indeed let $f$ be as in 1.10$)$, where $f_{l}(\cdot) \in C^{2}\left(\mathbb{R}^{n} ; \mathbb{R}^{n}\right)$ and $U_{l} \subset \mathbb{R}$ for $l \in\{1, \ldots, m\}$. Then for all $t$ such that $\mathcal{H}_{x u}[t] f_{u}[t]$ is symmetric and for any $k, l \in\{1, \ldots, m\}$

$$
\begin{aligned}
0=\left(\mathcal{H}_{x u}[t] f_{u}[t]-f_{u}[t]^{*} \mathcal{H}_{x u}[t]^{*}\right)_{k, l} & =\sum_{s=1}^{n} \sum_{j=1}^{n} p_{j}(t) \frac{\partial f_{k, j}}{\partial x_{s}}[t] f_{l, s}[t]-\sum_{s=1}^{n} f_{k, s}[t] \sum_{j=1}^{n} p_{j}(t) \frac{\partial f_{l, j}}{\partial x_{s}}[t] \\
& =\sum_{j=1}^{n} p_{j}(t) \sum_{s=1}^{n}\left(\frac{\partial f_{k, j}}{\partial x_{s}}[t] f_{l, s}[t]-f_{k, s}[t] \frac{\partial f_{l, j}}{\partial x_{s}}[t]\right),
\end{aligned}
$$

where $f_{l, j}$ is the $j$-th element of $f_{l}$. Hence for any $k, l \in\{1, \ldots, m\}$,

$$
\left\langle p(t), \frac{\partial f_{k}}{\partial x}[t] f_{l}[t]-\frac{\partial f_{l}}{\partial x}[t] f_{k}[t]\right\rangle=\left\langle p(t),\left[f_{k}, f_{l}\right][t]\right\rangle=0 .
$$

Proof. of Theorem 3.1. It is enough to consider the case when $\left|\left\{t \in\left(t_{1}, t_{2}\right): \bar{u}(t) \in \operatorname{Int} U\right\}\right|>0$.

We will prove that the statement is true at all points of

$$
\mathcal{L}:=\left\{s \in\left(t_{1}, t_{2}\right): \quad s \text { is a Lebesgue point of }(\bar{u}(\cdot), R(\cdot)) \text { and } \bar{u}(s) \in \operatorname{Int} U\right\},
$$

that is for almost every $t \in\left[t_{1}, t_{2}\right]$ such that $\bar{u}(t) \in \operatorname{Int} U$.

Fix $\bar{t} \in \mathcal{L}$. Note that (ii) implies that $\mathcal{H}_{x u}[\cdot] f_{u}[\cdot]$ is continuous at $\bar{t}$. For all $\rho>0, \eta>0$ define $A_{\rho, \eta}=\{s \in \mathcal{L} \cap(\bar{t}-\eta, \bar{t}+\eta):|| R(s)-R(\bar{t}) \|<\rho\}$. It is not difficult to check that $\left|A_{\rho, \eta}\right|>0$. Denote by $\Upsilon$ the set of all points in $A_{\rho, \eta}$ having the Lebesgue density in $A_{\rho, \eta}$ equal to one and let $m^{*}(\cdot)$ be defined as at the end of Section 2 for $\mathcal{R}(\cdot)=\mathcal{H}_{x u}[\cdot] f_{u}[\cdot], l=m$. Consider $\tau_{\rho, \eta} \in \Upsilon$ such that $m^{*}\left(\tau_{\rho, \eta}\right)=\min _{t \in \Upsilon} m^{*}(t)$. Then $\tau_{\rho, \eta} \in A_{\rho, \eta}$. We claim that it is enough to prove that $\mathcal{H}_{x u}\left[\tau_{\rho, \eta}\right] f_{u}\left[\tau_{\rho, \eta}\right]$ is symmetric and $R\left(\tau_{\rho, \eta}\right) \leq 0$. Indeed, if this holds true, then we can construct a sequence $t_{i} \rightarrow \bar{t}$ such that $\mathcal{H}_{x u}\left[t_{i}\right] f_{u}\left[t_{i}\right]$ is symmetric, $R\left(t_{i}\right) \leq 0$ and $\lim _{i \rightarrow \infty} R\left(t_{i}\right)=R(\bar{t})$. Then $R(\bar{t}) \leq 0$ and, by assumption (ii), $\mathcal{H}_{x u}[\bar{t}] f_{u}[\bar{t}]$ is symmetric. 
Fix $\rho>0, \eta>0$ and let $\Upsilon$ be as above. Set $\tau=\tau_{\rho, \eta}$ and define $\delta_{\varepsilon}, E_{\varepsilon}, E_{\varepsilon}^{1}, E_{\varepsilon}^{2}$ as in (2.5), (2.7) and (2.8) for the function $a(\cdot)=(\bar{u}(\cdot), R(\cdot))$ and $t=\tau \in \Upsilon$. To simplify the notations we also set $m^{*}=m^{*}(\tau)$. Consider $Q_{1}[t] \in M\left(m^{*} \times n\right), Q_{2}[t] \in M\left(\left(m-m^{*}\right) \times n\right), B_{1}[t] \in M\left(n \times m^{*}\right)$, $B_{2}[t] \in M\left(n \times\left(m-m^{*}\right)\right)$ such that

$$
\mathcal{H}_{x u}[t]=\left[\begin{array}{l}
Q_{1}[t] \\
Q_{2}[t]
\end{array}\right] \quad f_{u}[t]=\left[B_{1}[t] B_{2}[t]\right] .
$$

Then, by the choice of $m^{*}, Q_{1}[t] B_{1}[t]=\left(Q_{1}[t] B_{1}[t]\right)^{*}$ for all $t \in \Upsilon$. If $m^{*}=m$, then the matrices $Q_{2}(t)$ and $B_{2}(t)$ are simply absent in the above partition. Observe that if $m^{*}<m$, then

$$
Q_{1}[\tau] B_{2}[\tau] \neq\left(Q_{2}[\tau] B_{1}[\tau]\right)^{*} .
$$

For any $u \in \mathbb{R}^{m}$, let $u^{1} \in \mathbb{R}^{m^{*}}, u^{2} \in \mathbb{R}^{m-m^{*}}$ be such that $u^{*}=\left(\left(u^{1}\right)^{*},\left(u^{2}\right)^{*}\right)$. Again, if $m^{*}=m$, then $u=u^{1}$. In the case of a vector function $u(\cdot)$ we will use the notations $u^{1}(\cdot), u^{2}(\cdot)$ for the same type of partition.

Note that for $\varepsilon>0$ small enough we have $\left(\tau-\delta_{\varepsilon}, \tau+\delta_{\varepsilon}\right) \subset\left(t_{1}, t_{2}\right)$ and $\bar{u}(t) \in$ Int $U$ for all $t \in \Upsilon \cap E_{\varepsilon}$. For any $\varepsilon>0$ and $u \in \mathbb{R}^{m} \backslash\{0\}$, let us define

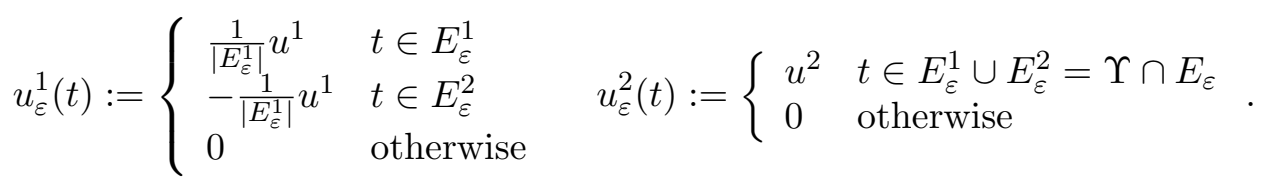

Observe that for all small $\varepsilon>0, u_{\varepsilon}(t) \neq 0$ if and only if $t \in \Upsilon \cap E_{\varepsilon}$. Moreover, by the maximum principle, $\mathcal{H}_{u}[t]=0$ for a.e. $t \in E_{\varepsilon}$, while for all $t \in[0,1] \backslash E_{\varepsilon}$ we have $0 \in T_{U}^{b(2)}(\bar{u}(t), 0)$. Therefore $u_{\varepsilon}(\cdot) \in \mathcal{U}_{A}$. Theorem 2.5 and assumption $(i)$ imply the following inequality

$$
-\frac{1}{2} y_{\varepsilon}(1)^{*} \varphi^{\prime \prime}(\bar{x}(1)) y_{\varepsilon}(1)+\frac{1}{2} \int_{0}^{1} y_{\varepsilon}(t)^{*} \mathcal{H}_{x x}[t] y_{\varepsilon}(t) d t+\int_{0}^{1} u_{\varepsilon}(t)^{*} \mathcal{H}_{x u}[t] y_{\varepsilon}(t) d t \leq 0,
$$

where $y_{\varepsilon}(\cdot)$ is the solution of $(2.1)$ for $u(\cdot)=u_{\varepsilon}(\cdot)$. Define for all $t \in[0,1]$

$$
\zeta_{\varepsilon}(t):=\int_{0}^{t} u_{\varepsilon}(s) d s
$$

Then, for all $t \in[0,1]$,

$$
\zeta_{\varepsilon}^{1}(t)=\frac{\left|E_{\varepsilon}^{1} \cap[0, t]\right|}{\left|E_{\varepsilon}^{1}\right|} u^{1}-\frac{\left|E_{\varepsilon}^{2} \cap[0, t]\right|}{\left|E_{\varepsilon}^{1}\right|} u^{1} \quad \text { and } \quad \zeta_{\varepsilon}^{2}(t)=u^{2}\left|\Upsilon \cap E_{\varepsilon} \cap[0, t]\right| .
$$

Thus $\zeta_{\varepsilon}(0)=0, \zeta_{\varepsilon}^{1}(t)=0$ for $t \in\left[0, \tau-\delta_{\varepsilon}\right] \cup\left[\tau+\delta_{\varepsilon}, 1\right]$ and $\left\|\zeta_{\varepsilon}^{1}(\cdot)\right\|_{\infty} \leq\left\|u^{1}\right\|$.

Moreover, by (2.9), for all $\varepsilon>0$ small enough

$$
\int_{0}^{1}\left\|\zeta_{\varepsilon}^{1}(t)\right\| d t=\int_{\tau-\delta_{\varepsilon}}^{\tau+\delta_{\varepsilon}}\left\|\zeta_{\varepsilon}^{1}(t)\right\| d t \leq \int_{\tau-\delta_{\varepsilon}}^{\tau+\delta_{\varepsilon}}\left\|u^{1}\right\| d t=2 \delta_{\varepsilon}\left\|u^{1}\right\| \leq 2\left|\Upsilon \cap E_{\varepsilon}\right| \cdot\left\|u^{1}\right\| .
$$

To simplify the notation we will omit for a while the dependence on $\varepsilon$. Integrating by parts

$$
\begin{aligned}
\int_{\tau-\delta}^{\tau+\delta} u(t)^{*} \mathcal{H}_{x u}[t] y(t) d t= & -\int_{\tau-\delta}^{\tau+\delta} \zeta(t)^{*} \frac{d}{d t}\left(\mathcal{H}_{x u} y\right)[t] d t+\left.\zeta(\cdot)^{*} \mathcal{H}_{x u}[\cdot] y(\cdot)\right|_{\tau-\delta} ^{\tau+\delta} \\
= & -\int_{\tau-\delta}^{\tau+\delta} \zeta(t)^{*} \frac{d}{d t} \mathcal{H}_{x u}[t] y(t) d t-\int_{\tau-\delta}^{\tau+\delta} \zeta(t)^{*} \mathcal{H}_{x u}[t] f_{x}[t] y(t) d t \\
& -\int_{\tau-\delta}^{\tau+\delta} \zeta(t)^{*} \mathcal{H}_{x u}[t] f_{u}[t] u(t) d t+\left.\zeta(\cdot)^{*} \mathcal{H}_{x u}[\cdot] y(\cdot)\right|_{\tau-\delta} ^{\tau+\delta}
\end{aligned}
$$


inequality $(3.3)$ becomes

$$
\begin{aligned}
& -\frac{1}{2} y(1)^{*} \varphi^{\prime \prime}(\bar{x}(1)) y(1)+\frac{1}{2} \int_{0}^{1} y(t)^{*} \mathcal{H}_{x x}[t] y(t) d t-\int_{\tau-\delta}^{\tau+\delta} \zeta(t)^{*} \frac{d}{d t} \mathcal{H}_{x u}[t] y(t) d t \\
& -\int_{\tau-\delta}^{\tau+\delta} \zeta(t)^{*} \mathcal{H}_{x u}[t] f_{x}[t] y(t) d t-\int_{\tau-\delta}^{\tau+\delta} \zeta(t)^{*} \mathcal{H}_{x u}[t] f_{u}[t] u(t) d t+\left.\zeta(\cdot)^{*} \mathcal{H}_{x u}[\cdot] y(\cdot)\right|_{\tau-\delta} ^{\tau+\delta} \leq 0 .
\end{aligned}
$$

Moreover

$$
\begin{aligned}
\int_{\tau-\delta}^{\tau+\delta} \zeta(t)^{*} \mathcal{H}_{x u}[t] f_{u}[t] u(t) d t= & \int_{\tau-\delta}^{\tau+\delta}\left(\zeta^{1}(t)^{*} Q_{1}[t] B_{1}[t] u^{1}(t)+\zeta^{1}(t)^{*} Q_{1}[t] B_{2}[t] u^{2}(t)\right. \\
& \left.+\zeta^{2}(t)^{*} Q_{2}[t] B_{1}[t] u^{1}(t)+\zeta^{2}(t)^{*} Q_{2}[t] B_{2}[t] u^{2}(t)\right) d t
\end{aligned}
$$

Integrating again by parts and using the symmetry of $Q_{1}[\cdot] B_{1}[\cdot]$ on $\Upsilon$ and that $\zeta^{1}(\tau \pm \delta)=0$ we obtain

$$
\begin{gathered}
-\int_{\tau-\delta}^{\tau+\delta} \zeta^{1}(t)^{*} Q_{1}[t] B_{1}[t] u^{1}(t) d t=\frac{1}{2} \int_{\tau-\delta}^{\tau+\delta} \zeta^{1}(t)^{*} \frac{d}{d t}\left(Q_{1} B_{1}\right)[t] \zeta^{1}(t) d t \\
-\int_{\tau-\delta}^{\tau+\delta} \zeta^{2}(t)^{*} Q_{2}[t] B_{1}[t] u^{1}(t) d t=\int_{\tau-\delta}^{\tau+\delta}\left(u^{2}(t)^{*} Q_{2}[t] B_{1}[t] \zeta^{1}(t)+\zeta^{2}(t)^{*} \frac{d}{d t}\left(Q_{2} B_{1}\right)[t] \zeta^{1}(t)\right) d t
\end{gathered}
$$

Then, coming back to (3.5), we have

$$
\begin{aligned}
0 \geq & -\frac{1}{2} y(1)^{*} \varphi^{\prime \prime}(\bar{x}(1)) y(1)+\frac{1}{2} \int_{0}^{1} y(t)^{*} \mathcal{H}_{x x}[t] y(t) d t \\
& -\int_{\tau-\delta}^{\tau+\delta} \zeta(t)^{*} \frac{d}{d t} \mathcal{H}_{x u}[t] y(t) d t-\int_{\tau-\delta}^{\tau+\delta} \zeta(t)^{*} \mathcal{H}_{x u}[t] f_{x}[t] y(t) d t+\left.\zeta(\cdot)^{*} \mathcal{H}_{x u}[\cdot] y(\cdot)\right|_{\tau-\delta} ^{\tau+\delta} \\
& +\frac{1}{2} \int_{\tau-\delta}^{\tau+\delta} \zeta^{1}(t)^{*} \frac{d}{d t}\left(Q_{1} B_{1}\right)[t] \zeta^{1}(t) d t-\int_{\tau-\delta}^{\tau+\delta} \zeta^{2}(t)^{*} Q_{2}[t] B_{2}[t] u^{2}(t) d t \\
& -\int_{\tau-\delta}^{\tau+\delta} \zeta^{1}(t)^{*}\left(Q_{1}[t] B_{2}[t]-\left(Q_{2}[t] B_{1}[t]\right)^{*}\right) u^{2}(t) d t+\int_{\tau-\delta}^{\tau+\delta} \zeta^{2}(t)^{*} \frac{d}{d t}\left(Q_{2} B_{1}\right)[t] \zeta^{1}(t) d t .
\end{aligned}
$$

Denote by $X(\cdot)$ the matrix solution of $X^{\prime}=f_{x}[t] X, X(0)=I d$. Integrating by parts we get

$$
\begin{aligned}
y(t) & =X(t) \int_{0}^{t} X^{-1}(s)\left(B_{1}[s] u^{1}(s)+B_{2}[s] u^{2}(s)\right) d s=X(t)\left[X^{-1}(\cdot) B_{1}[\cdot] \zeta^{1}(\cdot)\right]_{0}^{t} \\
& -X(t) \int_{0}^{t} \frac{d}{d s}\left(X^{-1} B_{1}\right)[s] \zeta^{1}(s) d s+X(t) \int_{0}^{t} X^{-1}(s) B_{2}[s] u^{2}(s) d s=B_{1}[t] \zeta^{1}(t) \\
& +X(t) \int_{0}^{t} X^{-1}(s)\left(f_{x}[s] B_{1}[s]-\frac{d}{d s} B_{1}[s]\right) \zeta^{1}(s) d s+X(t) \int_{0}^{t} X^{-1}(s) B_{2}[s] u^{2}(s) d s \\
& =\xi(t)+B_{1}[t] \zeta^{1}(t),
\end{aligned}
$$

where

$$
\xi(t):=X(t) \int_{0}^{t} X^{-1}(s)\left(f_{x}[s] B_{1}[s]-\frac{d}{d s} B_{1}[s]\right) \zeta^{1}(s) d s+X(t) \int_{0}^{t} X^{-1}(s) B_{2}[s] u^{2}(s) d s .
$$

The transformation $y(t)=\xi(t)+B_{1}[t] \zeta^{1}(t)$ is known as the Goh transformation. Observe that

$$
\xi^{\prime}(t)=f_{x}[t] \xi(t)+\left(f_{x}[t] B_{1}[t]-\frac{d}{d t} B_{1}[t]\right) \zeta^{1}(t)+B_{2}[t] u^{2}(t) \quad \xi(0)=0 .
$$


Substituting $y(\cdot)$ with $\xi(\cdot)+B_{1}[\cdot] \zeta^{1}(\cdot)$ in 3.6 we obtain

$$
\begin{aligned}
- & \frac{1}{2} \xi(1)^{*} \varphi^{\prime \prime}(\bar{x}(1)) \xi(1)+\frac{1}{2} \int_{0}^{1} \xi(t)^{*} \mathcal{H}_{x x}[t] \xi(t) d t+\int_{\tau-\delta}^{\tau+\delta} \zeta^{1}(t)^{*} B_{1}[t]^{*} \mathcal{H}_{x x}[t] \xi(t) d t \\
& -\int_{\tau-\delta}^{\tau+\delta} \zeta(t)^{*}\left(\frac{d}{d t} \mathcal{H}_{x u}[t]+\mathcal{H}_{x u}[t] f_{x}[t]\right) \xi(t) d t-\int_{\tau-\delta}^{\tau+\delta} \zeta^{2}(t)^{*} Q_{2}[t] B_{2}[t] u^{2}(t) d t \\
& -\int_{\tau-\delta}^{\tau+\delta} \zeta^{2}(t)^{*}\left(\frac{d}{d t} Q_{2}[t] B_{1}[t]+Q_{2}[t] f_{x}[t] B_{1}[t]-\frac{d}{d t}\left(Q_{2} B_{1}\right)[t]\right) \zeta^{1}(t) d t \\
& +\int_{\tau-\delta}^{\tau+\delta} \zeta^{1}(t)^{*}\left(\frac{1}{2} B_{1}[t]^{*} \mathcal{H}_{x x}[t] B_{1}[t]-\frac{d}{d t} Q_{1}[t] B_{1}[t]-Q_{1}[t] f_{x}[t] B_{1}[t]\right) \zeta^{1}(t) d t \\
& +\frac{1}{2} \int_{\tau-\delta}^{\tau+\delta} \zeta^{1}(t)^{*} \frac{d}{d t}\left(Q_{1} B_{1}\right)[t] \zeta^{1}(t) d t-\int_{\tau-\delta}^{\tau+\delta} \zeta^{1}(t)^{*}\left(Q_{1}[t] B_{2}[t]-\left(Q_{2}[t] B_{1}[t]\right)^{*}\right) u^{2}(t) d t \\
& +\left.\zeta(\cdot)^{*} \mathcal{H}_{x u}[\cdot] \xi(\cdot)\right|_{\tau-\delta} ^{\tau+\delta} \leq 0 .
\end{aligned}
$$

Restoring the subscript $\varepsilon$, by (3.4) and assumption (iii), $\left\|\xi_{\varepsilon}(\cdot)\right\|_{\infty}=O\left(\left|\Upsilon \cap E_{\varepsilon}\right|\right)$. Furthermore, by (iii),

$$
\begin{aligned}
& -\frac{1}{2} \xi_{\varepsilon}(1)^{*} \varphi^{\prime \prime}(\bar{x}(1)) \xi_{\varepsilon}(1)=o\left(\left|\Upsilon \cap E_{\varepsilon}\right|\right), \quad \int_{0}^{1} \xi_{\varepsilon}(t)^{*} \mathcal{H}_{x x}[t] \xi_{\varepsilon}(t) d t=o\left(\left|\Upsilon \cap E_{\varepsilon}\right|\right), \\
& \int_{\tau-\delta_{\varepsilon}}^{\tau+\delta_{\varepsilon}} \zeta_{\varepsilon}^{1}(t)^{*} B_{1}[t]^{*} \mathcal{H}_{x x}[t] \xi_{\varepsilon}(t) d t=o\left(\left|\Upsilon \cap E_{\varepsilon}\right|\right), \quad \int_{\tau-\delta_{\varepsilon}}^{\tau+\delta_{\varepsilon}} \zeta_{\varepsilon}^{2}(t)^{*} Q_{2}[t] B_{2}[t] u_{\varepsilon}^{2}(t)=o\left(\left|\Upsilon \cap E_{\varepsilon}\right|\right), \\
& \int_{\tau-\delta_{\varepsilon}}^{\tau+\delta_{\varepsilon}} \zeta_{\varepsilon}(t)^{*}\left(\frac{d}{d t} \mathcal{H}_{x u}[t]+\mathcal{H}_{x u}[t] f_{x}[t]\right) \xi_{\varepsilon}(t) d t=o\left(\left|\Upsilon \cap E_{\varepsilon}\right|\right), \\
& \int_{\tau-\delta_{\varepsilon}}^{\tau+\delta_{\varepsilon}} \zeta_{\varepsilon}^{2}(t)^{*}\left(-\frac{d}{d t} Q_{2}[t] B_{1}[t]-Q_{2}[t] f_{x}[t] B_{1}[t]+\frac{d}{d t}\left(Q_{2} B_{1}\right)[t]\right) \zeta_{\varepsilon}^{1}(t) d t=o\left(\left|\Upsilon \cap E_{\varepsilon}\right|\right)
\end{aligned}
$$

and

$$
\left.\zeta_{\varepsilon}(\cdot)^{*} \mathcal{H}_{x u}[\cdot] \xi_{\varepsilon}(\cdot)\right|_{\tau-\delta_{\varepsilon}} ^{\tau+\delta_{\varepsilon}}=o\left(\left|\Upsilon \cap E_{\varepsilon}\right|\right) .
$$

Since $\tau \in \mathcal{L}$, by $(2.9)$ and definition of $\delta_{\varepsilon}$, for some constant $c>0$ we have

$$
\begin{aligned}
& \int_{\tau-\delta_{\varepsilon}}^{\tau+\delta_{\varepsilon}} \zeta_{\varepsilon}^{1}(t)^{*}\left(\frac{1}{2} B_{1}[t]^{*} \mathcal{H}_{x x}[t] B_{1}[t]-\frac{1}{2} \frac{d}{d t} Q_{1}[t] B_{1}[t]-Q_{1}[t] f_{x}[t] B_{1}[t]+\frac{1}{2} Q_{1}[t] \frac{d}{d t} B_{1}[t]\right) \zeta_{\varepsilon}^{1}(t) d t= \\
& \int_{\tau-\delta_{\varepsilon}}^{\tau+\delta_{\varepsilon}} \zeta_{\varepsilon}^{1}(t)^{*}\left(\frac{1}{2} B_{1}[\tau]^{*} \mathcal{H}_{x x}[\tau] B_{1}[\tau]-\frac{1}{2} \frac{d}{d t} Q_{1}[\tau] B_{1}[\tau]-Q_{1}[\tau] f_{x}[\tau] B_{1}[\tau]+\frac{1}{2} Q_{1}[\tau] \frac{d}{d t} B_{1}[\tau]\right) \zeta_{\varepsilon}^{1}(t) d t \\
& +a_{\varepsilon}
\end{aligned}
$$

where $\left\|a_{\varepsilon}\right\| \leq c\left\|u^{1}\right\|^{2} \varepsilon^{2} 2 \delta_{\varepsilon}=o\left(\left|\Upsilon \cap E_{\varepsilon}\right|\right)$ and

$$
\begin{aligned}
-\int_{\tau-\delta_{\varepsilon}}^{\tau+\delta_{\varepsilon}} \zeta_{\varepsilon}^{1}(t)^{*}\left(Q_{1}[t] B_{2}[t]\right. & \left.-\left(Q_{2}[t] B_{1}[t]\right)^{*}\right) u_{\varepsilon}^{2}(t) d t= \\
& -\int_{\tau-\delta_{\varepsilon}}^{\tau+\delta_{\varepsilon}} \zeta_{\varepsilon}^{1}(t)^{*}\left(Q_{1}[\tau] B_{2}[\tau]-\left(Q_{2}[\tau] B_{1}[\tau]\right)^{*}\right) u_{\varepsilon}^{2}(t) d t+b_{\varepsilon},
\end{aligned}
$$

where $\left\|b_{\varepsilon}\right\| \leq c\left\|u^{1}\right\|\left\|u^{2}\right\| \varepsilon^{2} 2 \delta_{\varepsilon}=o\left(\left|\Upsilon \cap E_{\varepsilon}\right|\right)$. 
Therefore, applying Lemma 2.6 to $a(\cdot)=(\bar{u}(\cdot), R(\cdot))$, dividing by $\left|\Upsilon \cap E_{\varepsilon}\right|$ in (3.7) and passing to the limit when $\varepsilon \rightarrow 0+$, we obtain

$$
\begin{aligned}
& \frac{1}{3}\left(u^{1}\right)^{*}\left(\frac{1}{2} B_{1}[\tau]^{*} \mathcal{H}_{x x}[\tau] B_{1}[\tau]-\frac{1}{2} \frac{d}{d t} Q_{1}[\tau] B_{1}[\tau]-Q_{1}[\tau] f_{x}[\tau] B_{1}[\tau]+\frac{1}{2} Q_{1}[\tau] \frac{d}{d t} B_{1}[\tau]\right) u^{1} \\
& -\frac{1}{2}\left(u^{1}\right)^{*}\left(Q_{1}[\tau] B_{2}[\tau]-\left(Q_{2}[\tau] B_{1}[\tau]\right)^{*}\right) u^{2} \leq 0 .
\end{aligned}
$$

We claim that if $m^{*}<m$, then

$$
-\left(u^{1}\right)^{*}\left(Q_{1}[\tau] B_{2}[\tau]-\left(Q_{2}[\tau] B_{1}[\tau]\right)^{*}\right) u^{2} \leq 0
$$

Indeed, for $\lambda>0$ we have that $\left(\lambda\left(u^{1}\right)^{*},\left(u^{2}\right)^{*}\right)^{*} \in \mathbb{R}^{m}$. Thus, replacing $u^{1}$ by $\lambda u^{1}$ in $(3.8)$, dividing by $\lambda$ and letting $\lambda \rightarrow 0$, we get $(3.9)$. Furthermore, since $\left(-\left(u^{1}\right)^{*},\left(u^{2}\right)^{*}\right)^{*} \in \mathbb{R}^{m}$, we deduce that $\left(u^{1}\right)^{*}\left(Q_{1}[\tau] B_{2}[\tau]-\left(Q_{2}[\tau] B_{1}[\tau]\right)^{*}\right) u^{2} \leq 0$. Consequently

$$
\left(u^{1}\right)^{*}\left(Q_{1}[\tau] B_{2}[\tau]-\left(Q_{2}[\tau] B_{1}[\tau]\right)^{*}\right) u^{2}=0
$$

But $\left(\left(u^{1}\right)^{*},\left(u^{2}\right)^{*}\right)^{*}$ being arbitrary in $\mathbb{R}^{m}$, we conclude that $Q_{1}[\tau] B_{2}[\tau]-\left(Q_{2}[\tau] B_{1}[\tau]\right)^{*}=0$ contradicting (3.2). Thus $m^{*}=m$ and $B_{1}(\tau)=f_{u}[\tau], Q_{1}(\tau)=\mathcal{H}_{x u}[\tau]$. From (3.8) we deduce that $R(\tau) \leq 0$.

\section{SECOND ORDER CONDITIONS ON CONVEX POLYTOPES}

In this section $U \subset \mathbb{R}^{m}$ is a convex polytope in $\mathbb{R}^{m}$, i.e. $U$ is a finite intersection of affine half-spaces of dimension less or equal than $m$. Denote by $U_{0}$ the set of vertexes of $U$. Since $U$ is convex, its adjacent tangent cone $T_{U}^{b}\left(u_{0}\right)$ coincides with the tangent cone of convex analysis for any $u_{0} \in U$. Furthermore for every $u_{0} \in U, T_{U}^{b}\left(u_{0}\right)$ contains a subspace $P_{u_{0}}$ of maximal dimension $0 \leq r \leq m$. Moreover if $u_{0} \in \partial U$, then $r<m$ and for some $\nu>0$ and $h_{0}>0$ we have

$$
z+h u \in U, \forall u \in P_{u_{0}} \cap B, h \in\left[0, h_{0}\right], z \in U \text { with }\left\|z-u_{0}\right\| \leq \nu .
$$

In particular this implies that for any $u_{0} \in U$ there exists $\nu>0$ such that

$$
P_{u_{0}} \subset P_{z}, \quad \forall z \in U \text { with }\left\|z-u_{0}\right\| \leq \nu .
$$

Let $t \in[0,1]$ be such that $\bar{u}(t) \in \partial U \backslash U_{0}$ and $0<r<m$ be the dimension of the subspace $P_{\bar{u}(t)}$. Call $v_{1}, \ldots, v_{r}$ an orthonormal basis of $P_{\bar{u}(t)}$ and let $v_{r+1}, \ldots, v_{m}$ be such that $v_{1}, \ldots, v_{m}$ is an orthonormal basis of $\mathbb{R}^{m}$. Let $V(t) \in M(m \times m)$ be the change of basis matrix, i.e. $v_{i}=V(t) e_{i}$ for all $i \in\{1, \ldots, m\}$, where $\left\{e_{i}\right\}$ is the canonical basis of $\mathbb{R}^{m}$. Then $u \in P_{\bar{u}(t)}$ if and only if there exists $\tilde{u} \in \mathbb{R}^{r} \times\{0\}^{m-r}$ such that $u=V(t) \tilde{u}$. Consider $Q_{1}[t] \in M(r \times m)$, $Q_{3}[t] \in M((m-r) \times m), B_{1}[t] \in M(m \times r)$ and $B_{3}[t] \in M(m \times(m-r))$ such that

$$
V(t)^{*} \mathcal{H}_{x u}[t]=\left[\begin{array}{l}
Q_{1}[t] \\
Q_{3}[t]
\end{array}\right] \text { and } f_{u}[t] V(t)=\left[B_{1}[t] B_{3}[t]\right]
$$

Lemma 4.1. Let $(\bar{x}(\cdot), \bar{u}(\cdot))$ be a strong local minimizer of $(1.3)$ and $p(\cdot)$ be as in the maximum principle of Theorem 2.3. Assume that for some $0 \leq t_{1}<t_{2} \leq 1, \bar{u}(\cdot)$ is integrable on $\left[t_{1}, t_{2}\right]$ and that (3.1) holds true. Then, for a.e. $t \in\left[t_{1}, t_{2}\right]$ such that $\bar{u}(t) \in \partial U \backslash U_{0}$ and for $Q_{1}[t], B_{1}[t]$ defined as above, the matrix $Q_{1}[t] B_{1}[t]$ is symmetric and

$$
R_{1}(t):=\frac{1}{2} B_{1}[t]^{*} \mathcal{H}_{x x}[t] B_{1}[t]-\frac{1}{2} \frac{d}{d t} Q_{1}[t] B_{1}[t]-Q_{1}[t] f_{x}[t] B_{1}[t]+\frac{1}{2} Q_{1}[t] \frac{d}{d t} B_{1}[t]
$$

is negative semi-definite on $\mathbb{R}^{r}$.

Proof. It is enough to consider the case when $\left|\left\{t \in\left(t_{1}, t_{2}\right): \bar{u}(t) \in \partial U \backslash U_{0}\right\}\right|>0$. Let $R(\cdot)$ be defined as in Theorem 3.1. We prove the statements for all the points in

$$
\mathcal{L}:=\left\{s \in\left(t_{1}, t_{2}\right): s \text { is a Lebesgue point of }(\bar{u}(\cdot), R(\cdot)), \bar{u}(s) \in \partial U \backslash U_{0}\right\},
$$

that is for almost every $t \in[0,1]$ such that $\bar{u}(t) \in \partial U \backslash U_{0}$. 
Fix $\bar{t} \in \mathcal{L}$ and set $P=P_{\bar{u}(\bar{t})}$. By (ii), $\mathcal{H}_{x u}[\cdot] f_{u}[\cdot]$ is continuous at $\bar{t}$. For all $\rho>0, \eta>0$ define $A_{\rho, \eta}=\{s \in \mathcal{L} \cap(\bar{t}-\eta, \bar{t}+\eta):\|\bar{u}(s)-\bar{u}(\bar{t})\|+\|R(s)-R(\bar{t})\|<\rho\}$. Then $\left|A_{\rho, \eta}\right|>0$. Let $\nu>0$ and $h_{0}>0$ be such that (4.1) and (4.2) hold true with $u_{0}=\bar{u}(\bar{t})$. Below we restrict our attention to $0<\rho<\nu / 2$.

Consider $r>0$ and matrices $Q_{1}[\bar{t}], Q_{3}[\bar{t}], B_{1}[\bar{t}], B_{3}[\bar{t}], V(\bar{t})$ as described just before the statement of the lemma for $t=\bar{t}$. Set $V=V(\bar{t})$ and let $D_{1}[t] \in M(r \times m), D_{3}[t] \in M((m-r) \times m)$, $C_{1}[t] \in M(m \times r)$ and $C_{3}[t] \in M(m \times(m-r))$ be such that

$$
V^{*} \mathcal{H}_{x u}[t]=\left[\begin{array}{c}
D_{1}[t] \\
D_{3}[t]
\end{array}\right] \text { and } f_{u}[t] V=\left[C_{1}[t] C_{3}[t]\right]
$$

Define

$$
\Phi(t):=\frac{1}{2} C_{1}[t]^{*} \mathcal{H}_{x x}[t] C_{1}[t]-\frac{1}{2} \frac{d}{d t} D_{1}[t] C_{1}[t]-D_{1}[t] f_{x}[t] C_{1}[t]+\frac{1}{2} D_{1}[t] \frac{d}{d t} C_{1}[t] .
$$

Denote by $\Upsilon$ the set of all points in $A_{\rho, \eta}$ having the Lebesgue density in $A_{\rho, \eta}$ equal to one and let $m^{*}(\cdot)$ be defined as at the end of Section 2 for $\mathcal{R}(\cdot)=D_{1}[\cdot] C_{1}[\cdot], l=r$. Consider $\tau_{\rho, \eta} \in \Upsilon$ such that $m^{*}\left(\tau_{\rho, \eta}\right)=\min _{t \in \Upsilon} m^{*}(t)$. It is sufficient to prove that $D_{1}\left[\tau_{\rho, \eta}\right] C_{1}\left[\tau_{\rho, \eta}\right]$ is symmetric and $\Phi\left(\tau_{\rho, \eta}\right) \leq 0$. Indeed, this would imply the existence of $t_{i} \rightarrow \bar{t}$ such that $D_{1}\left[t_{i}\right] C_{1}\left[t_{i}\right]$ are symmetric, $\Phi\left(t_{i}\right) \leq 0$ and $\lim _{i \rightarrow \infty} \Phi\left(t_{i}\right)=R_{1}(\bar{t})$. Then $R_{1}(\bar{t}) \leq 0$ and, by assumption (ii), $Q_{1}[\bar{t}] B_{1}[\bar{t}]$ is symmetric.

Fix sufficiently small $\rho>0, \eta>0$ and let $\Upsilon$ be as above. Set $\tau=\tau_{\rho, \eta}, m^{*}=m^{*}(\tau)$ and define $\delta_{\varepsilon}, E_{\varepsilon}, E_{\varepsilon}^{1}, E_{\varepsilon}^{2}$ as in 2.5, 2.7) and 2.8 for the function $a(\cdot)=(\bar{u}(\cdot), R(\cdot))$ and $t=\tau \in \Upsilon$. Let $\tilde{Q}_{1}[t] \in M\left(m^{*} \times m\right), Q_{2}[t] \in M\left(\left(r-m^{*}\right) \times m\right), \tilde{B}_{1}[t] \in M\left(m \times m^{*}\right)$ and $\tilde{B}_{2}[t] \in M\left(m \times\left(r-m^{*}\right)\right)$ be such that

$$
V^{*} \mathcal{H}_{x u}[t]=\left[\begin{array}{c}
\tilde{Q}_{1}[t] \\
\tilde{Q}_{2}[t] \\
D_{3}[t]
\end{array}\right] \quad f_{u}[t] V=\left[\tilde{B}_{1}[t] \tilde{B}_{2}[t] C_{3}[t]\right] .
$$

Then $\tilde{Q}_{1}[t] \tilde{B}_{1}[t]=\left(\tilde{Q}_{1}[t] \tilde{B}_{1}[t]\right)^{*}$ for all $t \in \Upsilon$. If $m^{*}=r$, then $\tilde{Q}_{2}$ and $\tilde{B}_{2}$ are absent in the above partition. Moreover, by the definition of $m^{*}$, if $m^{*}<r$, then

$$
\left.\tilde{Q}_{1}[\tau] \tilde{B}_{2}[\tau] \neq\left(\tilde{Q}_{2}[\tau] \tilde{B}_{1}[\tau]\right)^{*}\right) \text {. }
$$

Let $\tilde{u}_{1} \in \mathbb{R}^{m^{*}}$ and $\tilde{u}_{2} \in \mathbb{R}^{r-m^{*}}$ be such $\left(\tilde{u}_{1}, \tilde{u}_{2}\right) \neq 0$. For any $\varepsilon>0$ define

$$
\tilde{u}_{\varepsilon}^{1}(t):=\left\{\begin{array}{ll}
\frac{1}{\left|E_{\varepsilon}^{1}\right|} \tilde{u}^{1} & t \in E_{\varepsilon}^{1} \\
-\frac{1}{\left|E_{\varepsilon}^{1}\right|} \tilde{u}^{1} & t \in E_{\varepsilon}^{2} \\
0 & \text { otherwise }
\end{array} \quad \tilde{u}_{\varepsilon}^{2}(t):=\left\{\begin{array}{ll}
\tilde{u}^{2} & t \in E_{\varepsilon}^{1} \cup E_{\varepsilon}^{2} \\
0 & \text { otherwise }
\end{array} \quad, \quad \tilde{u}_{\varepsilon}^{3}(t):=0 \in \mathbb{R}^{m-r}\right.\right.
$$

and $\tilde{u}_{\varepsilon}(t):=\left(\tilde{u}_{\varepsilon}^{1}(t), \tilde{u}_{\varepsilon}^{2}(t), \tilde{u}_{\varepsilon}^{3}(t)\right)$. Then $V \tilde{u}_{\varepsilon}(\cdot) \in \mathcal{U}_{A}$. Moreover, $0 \in T_{U}^{b(2)}\left(\bar{u}(t), V \tilde{u}_{\varepsilon}(t)\right)$ for a.e. $t \in\left[t_{1}, t_{2}\right]$. Thanks to Lemma 2.4 and Theorem 2.5 we obtain

$$
-\frac{1}{2} y_{\varepsilon}(1)^{*} \varphi^{\prime \prime}(\bar{x}(1)) y_{\varepsilon}(1)+\frac{1}{2} \int_{0}^{1} y_{\varepsilon}(t)^{*} \mathcal{H}_{x x}[t] y_{\varepsilon}(t) d t+\int_{0}^{1} \tilde{u}_{\varepsilon}(t)^{*} V^{*} \mathcal{H}_{x u}[t] y_{\varepsilon}(t) d t \leq 0,
$$

where $y_{\varepsilon}(\cdot)$ is the solution of (2.1) for $u(\cdot)=V \tilde{u}_{\varepsilon}(t)$.

Proceeding exactly in the same way as in the proof of Theorem 3.1, we obtain

$$
\begin{aligned}
& \frac{1}{3}\left(\tilde{u}^{1}\right)^{*}\left(\frac{1}{2} \tilde{B}_{1}[\tau]^{*} \mathcal{H}_{x x}[\tau] \tilde{B}_{1}[\tau]-\frac{1}{2} \frac{d}{d t} \tilde{Q}_{1}[\tau] \tilde{B}_{1}[\tau]-\tilde{Q}_{1}[\tau] f_{x}[\tau] \tilde{B}_{1}[\tau]+\frac{1}{2} \tilde{Q}_{1}[\tau] \frac{d}{d t} \tilde{B}_{1}[\tau]\right) \tilde{u}^{1} \\
& -\frac{1}{2}\left(\tilde{u}^{1}\right)^{*}\left(\tilde{Q}_{1}[\tau] \tilde{B}_{2}[\tau]-\left(\tilde{Q}_{2}[\tau] \tilde{B}_{1}[\tau]\right)^{*}\right) \tilde{u}^{2} \leq 0 .
\end{aligned}
$$

If $m^{*}<r$, then, by the same reasonings as at the end of the proof of Theorem 3.1, we get $\tilde{Q}_{1}[\tau] \tilde{B}_{2}[\tau]=\left(\tilde{Q}_{2}[\tau] \tilde{B}_{1}[\tau]\right)^{*}$ contradicting $(4.3)$. Thus $m^{*}=r$ and we deduce from (4.4) that $R_{1}(\tau) \leq 0$ on $\mathbb{R}^{r}$. 
Remark 4.2. The matrices $Q_{1}[t]$ and $B_{1}[t]$ depend on the choice of the basis for $P_{\bar{u}(t)}$. However, the above lemma implies that for almost all $t \in\left[t_{1}, t_{2}\right]$ such that $\bar{u}(t) \in \partial U \backslash U_{0}, \mathcal{H}_{x u}[t] f_{u}[t]$ is symmetric on the subspace $P_{\bar{u}(t)}$ and $R(t)$ is negative semi-definite on $P_{\bar{u}(t)}$.

Theorem 4.3. Let $(\bar{x}(\cdot), \bar{u}(\cdot))$ be a strong local minimizer for the Mayer problem (1.3) and $p(\cdot)$ be as in the maximum principle of Theorem 2.3. Assume that for some $0 \leq t_{1}<t_{2} \leq 1, \bar{u}(\cdot)$ is integrable on $\left[t_{1}, t_{2}\right]$ and (3.1) holds true. Then for a.e. $t \in\left[t_{1}, t_{2}\right], \mathcal{H}_{x u}[t] f_{u}[t]$ is symmetric on the subspace $P_{\bar{u}(t)}$ and $R(t)$ is negative semi-definite on $P_{\bar{u}(t)}$.

Proof. Consider $A_{1}=\left\{t \in\left[t_{1}, t_{2}\right]: u(t) \in U_{0}\right\}, A_{2}=\left\{t \in\left[t_{1}, t_{2}\right]: u(t) \in \partial U \backslash U_{0}\right\}, A_{3}=\{t \in$ $\left.\left[t_{1}, t_{2}\right]: u(t) \in \operatorname{Int} U\right\}$. Since for every $t \in A_{1}, P_{\bar{u}(t)}=0$, the statement of theorem holds true on $A_{1}$. By Remark 4.2 it holds true for a.e. $t \in A_{2}$. Theorem 3.1 completes the proof.

Corollary 4.4. Under all the assumptions of Theorem 4.3 suppose that $f$ is as in $(1.10)$, where $f_{l}(\cdot) \in C^{2}\left(\mathbb{R}^{n} ; \mathbb{R}^{n}\right)$ and $U_{l} \subset \mathbb{R}$ is a closed (possibly unbounded) interval for all $l \in\{1, \ldots, m\}$. Consider the matrix valued mapping $\Theta:[0,1] \rightarrow M(m \times m)$, whose elements are

$$
\theta_{k, l}(t):=\left\langle p(t),\left[f_{k}, f_{l}\right](\bar{x}(t))\right\rangle \quad \forall k, l \in\{1, \ldots, m\} .
$$

Then for a.e. $t \in\left[t_{1}, t_{2}\right]$, the subspace $\Theta(t)\left(P_{\bar{u}(t)}\right)$ is orthogonal to $P_{\bar{u}(t)}$.

Proof. By Theorem 4.3 applied to the convex polytope $U=U_{1} \times \ldots \times U_{m}$, for a.e. $t \in\left[t_{1}, t_{2}\right]$, $\mathcal{H}_{x u}[t] f_{u}[t]$ is symmetric on $P_{\bar{u}(t)}$. Fix such $t$. Since $a^{*}\left(\mathcal{H}_{x u}[t] f_{u}[t]-\left(\mathcal{H}_{x u}[t] f_{u}[t]\right)^{*}\right) b=0$ for all $a, b \in P_{\bar{u}(t)}$, denoting by $f_{l, j}$ is the $j$-th element of $f_{l}$, we obtain

$$
\begin{aligned}
0 & =\sum_{k=1}^{m} \sum_{l=1}^{m} a_{k}\left(\mathcal{H}_{x u}[t] f_{u}[t]-f_{u}[t]^{*} \mathcal{H}_{x u}[t]^{*}\right)_{k, l} b_{l} \\
& =\sum_{k=1}^{m} \sum_{l=1}^{m} a_{k}\left(\sum_{s=1}^{n} \sum_{j=1}^{n} p_{j}(t) \frac{\partial f_{k, j}}{\partial x_{s}}(\bar{x}(t)) f_{l, s}(\bar{x}(t))-\sum_{s=1}^{n} f_{k, s}(\bar{x}(t)) \sum_{j=1}^{n} p_{j}(t) \frac{\partial f_{l, j}}{\partial x_{s}}(\bar{x}(t))\right) b_{l} \\
& =\sum_{k=1}^{m} \sum_{l=1}^{m} a_{k}\left(\sum_{j=1}^{n} p_{j}(t) \sum_{s=1}^{n}\left(\frac{\partial f_{k, j}}{\partial x_{s}}(\bar{x}(t)) f_{l, s}(\bar{x}(t))-f_{k, s}(\bar{x}(t)) \frac{\partial f_{l, j}}{\partial x_{s}}(\bar{x}(t))\right)\right) b_{l} .
\end{aligned}
$$

Hence

$$
\left\langle p(t), \sum_{k=1}^{m} \sum_{l=1}^{m} a_{k} \frac{\partial f_{k}}{\partial x}(\bar{x}(t)) f_{l}(\bar{x}(t))-\frac{\partial f_{l}}{\partial x}(\bar{x}(t)) f_{k}(\bar{x}(t)) b_{l}\right\rangle=\left\langle p(t), \sum_{k=1}^{m} \sum_{l=1}^{m} a_{k}\left[f_{k}, f_{l}\right](\bar{x}(t)) b_{l}\right\rangle=0
$$

and therefore $0=\sum_{k=1}^{m} \sum_{l=1}^{m} a_{k}\left\langle p(t),\left[f_{k}, f_{l}\right](\bar{x}(t))\right\rangle b_{l}=\langle a, \Theta(t) b\rangle$.

\section{Remark 4.5.}

a) The matrix $\Theta(t)$ of Corollary 4.4 is called in [9] the Goh matrix. Note that by Theorem 3.1 it is equal to zero for a.e. $t \in\left[t_{1}, t_{2}\right]$ such that $\bar{u}(t) \in \operatorname{Int}\left(U_{1} \times \ldots \times U_{m}\right)$.

b) Using the notations $\tilde{u}, \tilde{a}, \tilde{b}, r$ and $V(t)$ introduced right before Lemma 4.1, we can further develop the conclusions of the above corollary. Fix $t \in\left[t_{1}, t_{2}\right]$ such that $\mathcal{H}_{x u}[t] f_{u}[t]$ is symmetric on the subspace $P_{\bar{u}(t)}$. Then

$$
f(x, u)=f_{0}(x)+\sum_{l=1}^{m}\left(\sum_{j=1}^{m} V(t)_{l, j} \tilde{u}_{j}\right) f_{l}(x),
$$

and we can define

$$
\tilde{f}(x, \tilde{u}):=f(x, u)=f_{0}(x)+\sum_{l=1}^{r} \tilde{u}_{l} \tilde{f}_{l}(x)
$$


where $\tilde{f}_{l}(x):=\sum_{j=1}^{m} V(t)_{j, l} f_{j}(x)$ for $l \in\{1, \ldots, r\}$ (recall that $\tilde{u}_{l}=0$ for $l \in\{r+1, \ldots, m\}$ ). Note that $\tilde{f}_{\tilde{u}}[t]=f_{u}[t] V(t)$ and call $\tilde{\mathcal{H}}(x, \tilde{u}):=\langle p(t), \tilde{f}(x, \tilde{u})\rangle$. Now for any $a, b \in P_{\bar{u}(t)}$ we have

$$
a^{*}\left(\mathcal{H}_{x u}[t] f_{u}[t]-\left(\mathcal{H}_{x u}[t] f_{u}[t]\right)^{*}\right) b=\tilde{a}^{*} V(t)^{*}\left(\mathcal{H}_{x u}[t] f_{u}[t]-\left(\mathcal{H}_{x u}[t] f_{u}[t]\right)^{*}\right) V(t) \tilde{b}=0 .
$$

Therefore for any $k, l \in\{1, \ldots, r\}$

$$
\begin{aligned}
0 & =\left(V(t)^{*}\left(\mathcal{H}_{x u}[t] f_{u}[t]-f_{u}[t]^{*} \mathcal{H}_{x u}[t]^{*}\right) V(t)\right)_{k, l}=\left(\tilde{\mathcal{H}}_{x \tilde{u}}[t] \tilde{f}_{\tilde{u}}[t]-\tilde{f}_{\tilde{u}}[t]^{*} \tilde{\mathcal{H}}_{x \tilde{u}}[t]^{*}\right)_{k, l} \\
& =\sum_{s=1}^{n} \sum_{j=1}^{n} p_{j}(t) \frac{\partial \tilde{f}_{k, j}}{\partial x_{s}}[t] \tilde{f}_{l, s}[t]-\sum_{s=1}^{n} \tilde{f}_{k, s}[t] \sum_{j=1}^{n} p_{j}(t) \frac{\partial \tilde{f}_{l, j}}{\partial x_{s}}[t] \\
& =\sum_{j=1}^{n} p_{j}(t) \sum_{s=1}^{n}\left(\frac{\partial \tilde{f}_{k, j}}{\partial x_{s}}[t] \tilde{f}_{l, s}[t]-\tilde{f}_{k, s}[t] \frac{\partial \tilde{f}_{l, j}}{\partial x_{s}}[t]\right)=\left\langle p(t),\left[\tilde{f}_{k}, \tilde{f}_{l}\right][t]\right\rangle .
\end{aligned}
$$

That is $p(t)$ is orthogonal to the Lie bracket $\left[\tilde{f}_{k}, \tilde{f}_{l}\right](\bar{x}(t))$ for all $k, l \in\{1, \ldots, r\}$.

\section{SECOND ORDER CONDITIONS FOR SMOOTH CONTROL CONSTRAINTS}

In this section we consider a proper closed set $U \subset \mathbb{R}^{m}$ of class $C^{2}$ whose definition we recall now. For the unit ball $B$ in $\mathbb{R}^{m}$ let

$$
B_{0}:=\left\{v=\left(v_{1}, \ldots, v_{m}\right) \in B: v_{m}=0\right\} \quad \& \quad B_{+}:=\left\{v=\left(v_{1}, \ldots, v_{m}\right) \in B: v_{m}>0\right\} .
$$

Definition 5.1. $U$ is said to be of class $C^{2}$ if for each $u \in \partial U$ there exist

(i) a neighborhood $W(u)$ of $u$, and

(ii) a bijective map $g_{u}: W(u) \rightarrow B$ with the following properties:

$$
\begin{gathered}
g_{u}(\cdot) \in C^{2}(W(u) ; B), \quad h_{u}(\cdot):=g_{u}^{-1}(\cdot) \in C^{2}(B ; W(u)), \text { Int } U \cap W(u)=h_{u}\left(B_{+}\right), \\
\Gamma_{u}:=\partial U \cap W(u)=h_{u}\left(B_{0}\right), B_{0}=g_{u}\left(\Gamma_{u}\right) .
\end{gathered}
$$

Remark 5.2. If $U$ is of class $C^{2}$, then

a) For each $u_{0} \in \partial U$ there exists a neighborhood $W\left(u_{0}\right)$ of $u_{0}$ such that the oriented distance $b_{U}(\cdot) \in C^{2}\left(W\left(u_{0}\right)\right)$ and $\left\|\nabla b_{U}(u)\right\|=1$ for all $u \in W\left(u_{0}\right)$, see for instance Theorem 4.3 in [11];

b) For every $u_{0} \in \partial U, T_{U}^{b}\left(u_{0}\right)=\left\{v \in \mathbb{R}^{m}:\left\langle\nabla b_{U}\left(u_{0}\right), v\right\rangle \leq 0\right\}$ and the subspace

$$
P_{u_{0}}:=\left\{v \in \mathbb{R}^{m}:\left\langle\nabla b_{U}\left(u_{0}\right), v\right\rangle=0\right\}=\partial T_{U}^{b}\left(u_{0}\right)
$$

has the dimension equal to $m-1$.

c) For every $u_{0} \in \partial U, \nabla b_{U}\left(u_{0}\right)$ is the unit outward normal to $U$ at $u_{0}$ and, applying the inverse mapping theorem to $b_{U}$, it is not difficult to show that for any $u \in \partial T_{U}^{b}\left(u_{0}\right)$ we have $-\frac{1}{2}\left(u^{*} b_{U}^{\prime \prime}\left(u_{0}\right) u\right) \nabla b_{U}\left(u_{0}\right) \in T_{U}^{b(2)}\left(u_{0}, u\right)$.

Let $(\bar{x}(\cdot), \bar{u}(\cdot))$ be a strong local minimizer for the Mayer problem 1.3. Define the following matrix valued mapping $C(\cdot)$ on $[0,1]$

$$
C(t)=\left\{\begin{array}{l}
b_{U}^{\prime \prime}(\bar{u}(t)) \text { if } \bar{u}(t) \in \partial U \\
0 \text { otherwise }
\end{array}\right.
$$

Theorem 5.3. Let $(\bar{x}(\cdot), \bar{u}(\cdot))$ be a strong local minimizer for the Mayer problem (1.3) and $p(\cdot)$ be as in the maximum principle of Theorem 2.3. Then for every $u(\cdot) \in \mathcal{U}_{A}$ either $\mathcal{H}_{u}[t] u(t)<0$ on a set of positive measure or $\mathcal{H}_{u}[t] u(t)=0$ a.e. in $[0,1]$ and the inequality

$$
\begin{aligned}
0 \geq & \int_{0}^{1}\left(\frac{1}{2} y(t)^{*} \mathcal{H}_{x x}[t] y(t)+u(t)^{*} \mathcal{H}_{x u}[t] y(t)+\frac{1}{2} u(t)^{*}\left(\mathcal{H}_{u u}[t]-\left\|\mathcal{H}_{u}[t]\right\| C(t)\right) u(t)\right) d t \\
& -\frac{1}{2} y(1)^{*} \varphi^{\prime \prime}(\bar{x}(1)) y(1)
\end{aligned}
$$

holds true, where $y(\cdot)$ is the solution of (2.1). 
Proof. By the maximum principle, $\mathcal{H}_{u}[t]=\left\|\mathcal{H}_{u}[t]\right\| \nabla b_{U}(\bar{u}(t))$ for a.e. $t$ such that $\bar{u}(t) \in \partial U$. This, Remark 5.2 c) and Theorem 2.5 imply the claim.

For a trajectory/control pair $(\bar{x}(\cdot), \bar{u}(\cdot))$ assume that for some $0 \leq t_{1}<t_{2} \leq 1, \bar{u}(\cdot)$ is Lipschitz on $\left(t_{1}, t_{2}\right)$ and let $\bar{t} \in\left(t_{1}, t_{2}\right)$ be such that $\bar{u}(\bar{t}) \in \partial U$. Then there exists $\eta>0$ such that $[\bar{t}-\eta, \bar{t}+\eta] \subset\left(t_{1}, t_{2}\right)$ and $b_{U} \in C^{2}$ on a neighborhood of the set $\bar{u}([\bar{t}-\eta, \bar{t}+\eta])$. For every $t \in[\bar{t}-\eta, \bar{t}+\eta]$ define the $m-1$ dimensional subspace

$$
S(t):=\left\{u \in \mathbb{R}^{m}:\left\langle\nabla b_{u}(\bar{u}(t)), u\right\rangle=0\right\} .
$$

Remark 5.4. In the same way as in Lemma 2.4 it can be shown that $\mathcal{H}_{u}[t] u=0$ for any $u \in S(t)$ and a.e. $t \in(\bar{t}-\eta, \bar{t}+\eta)$. Hence (5.1) holds for every $u(\cdot) \in \mathcal{U}_{A}$, such that $u(t) \in S(t)$ a.e. in $(\bar{t}-\eta, \bar{t}+\eta)$ and $u(t)=0$ on $[0,1] \backslash(\bar{t}-\eta, \bar{t}+\eta)$.

For every $t \in(\bar{t}-\eta, \bar{t}+\eta)$ consider an orthonormal basis $\left\{v_{1}(t), \ldots, v_{m-1}(t)\right\}$ of $S(t)$. Thus $\left\{v_{1}(t), \ldots, v_{m-1}(t), \nabla b_{U}(\bar{u}(t))\right\}$ is an orthonormal basis for $\mathbb{R}^{m}$. Let $V(t) \in M(m \times m)$ be the change of basis matrix, i.e. $v_{i}(t)=V(t) e_{i}$ for all $i \in\{1, \ldots, m-1\}$ and $\nabla b_{U}(\bar{u}(t))=V(t) e_{m}$, where $\left\{e_{i}\right\}$ is the canonical basis of $\mathbb{R}^{m}$.

The matrix-valued function $V(\cdot)$ can be chosen on $(\bar{t}-\eta, \bar{t}+\eta)$ in such way that its regularity depends only on the regularity of $\bar{u}(\cdot)$. Indeed we have the following lemma.

Lemma 5.5. For all $\tilde{x} \in \mathbb{R}^{m} \backslash\{0\}$, there exist a neighborhood $W(\tilde{x}) \subset \mathbb{R}^{m}$ of $\tilde{x}$ and an analytic map $\mathcal{V}: W(\tilde{x}) \rightarrow M(m \times m)$ such that

$$
\mathcal{V}(x):=\left[x_{1}(x), \ldots, x_{m-1}(x), x\right],
$$

where $x_{1}(x), \ldots, x_{m-1}(x) \in \mathbb{R}^{m}$ are such that $\left\{x_{1}(x), \ldots, x_{m-1}(x)\right\}$ is an orthonormal basis of $\{x\}^{\perp}:=\left\{v \in \mathbb{R}^{m}:\langle v, x\rangle=0\right\}$.

Proof. Fix $\tilde{x} \in \mathbb{R}^{m}, \tilde{x} \neq 0$ and complete it to a basis $\left\{x_{1}(\tilde{x}), \ldots, x_{m-1}(\tilde{x}), \tilde{x}\right\}$ of $\mathbb{R}^{m}$. Then, there exists a neighborhood $W(\tilde{x})$ of $\tilde{x}$ such that $\operatorname{det}\left(x_{1}(\tilde{x}), \ldots, x_{m-1}(\tilde{x}), x\right) \neq 0$ for all $x \in W(\tilde{x})$ and therefore $\left\{x_{1}(\tilde{x}), \ldots, x_{m-1}(\tilde{x}), x\right\}$ is a basis of $\mathbb{R}^{m}$. Moreover the map $x \mapsto\left(x_{1}(\tilde{x}), \ldots, x_{m-1}(\tilde{x}), x\right)$ is analytic. Using the Gram-Schmidt process we can orthonormalize this basis getting the analytic map $\mathcal{V}(\cdot)$.

Define

$$
u_{0}(t):= \begin{cases}\frac{d}{d t} \bar{u}(t) & \text { if } \bar{u} \text { is differentiable at } t \in[\bar{t}-\eta, \bar{t}+\eta] \\ 0 & \text { otherwise. }\end{cases}
$$

and $v_{0}(t):=b_{U}^{\prime \prime}(\bar{u}(t)) u_{0}(t)$.

Since $b_{U}^{\prime \prime}(\bar{u}(\cdot))$ is continuous on $[\bar{t}-\eta, \bar{t}+\eta]$, every Lebesgue point of $u_{0}(\cdot)$ in $[\bar{t}-\eta, \bar{t}+\eta]$ is also a Lebesgue point of $v_{0}(\cdot)$ and if $\bar{u}$ is differentiable at $t \in[\bar{t}-\eta, \bar{t}+\eta]$, then

$$
\frac{d}{d t}\left(\nabla b_{U}(\bar{u}(\cdot))\right)(t)=v_{0}(t) .
$$

Thus $\eta>0$ and $V(\cdot)$ can be chosen in such way that $V(\cdot)$ is Lipschitz on $[\bar{t}-\eta, \bar{t}+\eta] \subset\left(t_{1}, t_{2}\right)$ and if $\bar{u}$ is differentiable at $t \in[\bar{t}-\eta, \bar{t}+\eta]$, then so is $V(\cdot)$ and every Lebesgue point $t \in[\bar{t}-\eta, \bar{t}+\eta]$ of $\frac{d}{d t} \bar{u}(\cdot)$ is also a Lebesgue point of $\frac{d}{d t} V(\cdot)$. Set $V(t)=0$ for all $t \in[0,1] \backslash[\bar{t}-\eta, \bar{t}+\eta]$.

For any $t \in[0,1]$ consider $Q_{1}[t] \in M((m-1) \times n), Q_{3}[t]^{*} \in \mathbb{R}^{n}, B_{1}[t] \in M(n \times(m-1))$ and $B_{3}[t] \in \mathbb{R}^{n}$ such that

$$
V(t)^{*} \mathcal{H}_{x u}[t]=\left[\begin{array}{c}
Q_{1}[t] \\
Q_{3}[t]
\end{array}\right] \text { and } f_{u}[t] V(t)=\left[B_{1}[t] B_{3}[t]\right]
$$


Lemma 5.6. Let $(\bar{x}(\cdot), \bar{u}(\cdot))$ be a strong local minimizer for the Mayer problem (1.3) and $p(\cdot)$ be as in the maximum principle of Theorem 2.3. Assume that for some $0 \leq t_{1}<t_{2} \leq 1, \bar{u}(\cdot)$ is Lipschitz on $\left[t_{1}, t_{2}\right], b_{U}(\cdot) \in C^{2}$ on a neighborhood of $\bar{u}\left(\left[t_{1}, t_{2}\right]\right)$ and

(i) $u^{*}\left(\mathcal{H}_{u u}[s]-\left\|\mathcal{H}_{u}[t]\right\| b_{U}^{\prime \prime}(\bar{u}(s))\right) u=0$ for all $u \in S(s)$ and for a.e. $s \in\left[t_{1}, t_{2}\right]$;

$\begin{cases}(i i) & \mathcal{H}_{x u}[\cdot], f_{u}[\cdot] \text { are Lipschitz on }\left[t_{1}, t_{2}\right] \\ \text { (iii) } & \mathcal{H}_{x x}[\cdot], f_{x}[\cdot] \text { are essentially bounded on }\left[t_{1}, t_{2}\right] .\end{cases}$

Then for almost every $\bar{t} \in\left(t_{1}, t_{2}\right)$ such that $\bar{u}(\bar{t}) \in \partial U$ there exist $\eta>0$ satisfying $(\bar{t}-\eta, \bar{t}+$ $\eta) \subset\left(t_{1}, t_{2}\right)$ and a Lipschitz change of basis $V(\cdot):(\bar{t}-\eta, \bar{t}+\eta) \rightarrow M(m \times m)$ such that for $Q_{i}[\cdot], B_{i}[\cdot], i=1,3$ defined as before the statement of the lemma, the matrix $Q_{1}[\bar{t}] B_{1}[\bar{t}]$ is symmetric and

$$
R_{1}(t):=\frac{1}{2} B_{1}[\bar{t}]^{*} \mathcal{H}_{x x}[\bar{t}] B_{1}[\bar{t}]-\frac{1}{2} \frac{d}{d t} Q_{1}[\bar{t}] B_{1}[\bar{t}]-Q_{1}[\bar{t}] f_{x}[\bar{t}] B_{1}[\bar{t}]+\frac{1}{2} Q_{1}[\bar{t}] \frac{d}{d t} B_{1}[\bar{t}]
$$

is negative semi-definite on $\mathbb{R}^{m-1}$.

Note that if $\mathcal{H}_{u}[\cdot]=0$ and $\mathcal{H}_{u u}[\cdot]=0$ a.e. in $\left[t_{1}, t_{2}\right]$, then the assumption $(i)$ holds true.

Proof. It is enough to consider the case when $\left|\left\{t \in\left(t_{1}, t_{2}\right): \bar{u}(t) \in \partial U\right\}\right|>0$.

Consider the matrix valued map $R_{2}(\cdot)$ on $\left(t_{1}, t_{2}\right)$ defined a.e. in $\left(t_{1}, t_{2}\right)$ by

$$
R_{2}(t):=\left(\frac{d}{d t} f_{u}[t], \frac{d}{d t} \mathcal{H}_{x u}[t], \mathcal{H}_{x x}[t], f_{x}[t]\right) .
$$

We will prove that the statement is true at all points of the set

$$
\mathcal{L}:=\left\{s \in\left(t_{1}, t_{2}\right): \bar{u}(s) \in \partial U \text { and } s \text { is a Lebesgue point of }\left(\frac{d}{d t} \bar{u}(\cdot), R_{2}(\cdot)\right)\right\},
$$

that is for almost every $t \in\left(t_{1}, t_{2}\right)$ such that $\bar{u}(t) \in \partial U$.

Fix such $\bar{t}$. By the comments preceding the statement of the lemma, there exist $\eta>0$ with $(\bar{t}-\eta, \bar{t}+\eta) \subset\left(t_{1}, t_{2}\right)$ and a Lipschitz change of basis $V(\cdot):(\bar{t}-\eta, \bar{t}+\eta) \rightarrow M(m \times m)$ such that if $\bar{u}(\cdot)$ is differentiable at $t \in[\bar{t}-\eta, \bar{t}+\eta]$, then so is $V(\cdot)$ and every Lebesgue point $t \in[\bar{t}-\eta, \bar{t}+\eta]$ of $\frac{d}{d t} \bar{u}(\cdot)$ is also a Lebesgue point of $\frac{d}{d t} V(\cdot)$. Let $Q_{i}[\cdot], B_{i}[\cdot], i=1,3$ be defined as before the statement of the lemma.

For all $\rho>0, \eta>0$ consider

$$
A_{\rho, \eta}:=\left\{s \in \mathcal{L} \cap(\bar{t}-\eta, \bar{t}+\eta):\left\|\frac{d}{d t} V(s)-\frac{d}{d t} V(\bar{t})\right\|+\left\|R_{2}(s)-R_{2}(\bar{t})\right\|<\rho\right\} .
$$

Then $\left|A_{\rho, \eta}\right|>0$. Denote by $\Upsilon$ the set of all points in $A_{\rho, \eta}$ having the Lebesgue density in $A_{\rho, \eta}$ equal to one and let $m^{*}(\cdot)$ be defined as at the end of Section 2 for $\mathcal{R}(\cdot)=Q_{1}[\cdot] B_{1}[\cdot], l=$ $m$. Consider $\tau_{\rho, \eta} \in \Upsilon$ such that $m^{*}\left(\tau_{\rho, \eta}\right)=\min _{t \in \Upsilon} m^{*}(t)$. It is enough to prove that for all small $\rho>0, \eta>0, Q_{1}\left[\tau_{\rho, \eta}\right] B_{1}\left[\tau_{\rho, \eta}\right]$ is symmetric and $R_{1}\left(\tau_{\rho, \eta}\right) \leq 0$. Indeed, if this holds true, then we can construct a sequence $t_{i} \rightarrow \bar{t}$ such that $Q_{1}\left[t_{i}\right] B_{1}\left[t_{i}\right]$ is symmetric, $R_{1}\left(t_{i}\right) \leq 0$ and $\lim _{i \rightarrow \infty} R_{1}\left(t_{i}\right)=R_{1}(\bar{t})$. Then $R_{1}(\bar{t}) \leq 0$ and, since $\mathcal{H}_{x u}[\cdot] f_{u}[\cdot]$ and $V(\cdot)$ are Lipschitz, $Q_{1}[\bar{t}] B_{1}[\bar{t}]$ is symmetric.

Fix $\rho>0, \eta>0$ sufficiently small and let $\Upsilon$ be as above. Set $\tau=\tau_{\rho, \eta}, m^{*}=m^{*}(\tau)$ and define $\delta_{\varepsilon}, E_{\varepsilon}, E_{\varepsilon}^{1}, E_{\varepsilon}^{2}$ as in 2.5, 2.7) and 2.8 for the function $a(\cdot)=\left(\frac{d}{d t} V(\cdot), R_{2}(\cdot)\right)$ and $t=\tau \in \Upsilon$.

Consider $\tilde{Q}_{1}[t] \in M\left(m^{*} \times n\right), \tilde{Q}_{2}[t] \in M\left(\left(m-1-m^{*}\right) \times n\right), \tilde{B}_{1}[t] \in M\left(n \times m^{*}\right)$ and $\tilde{B}_{2}[t] \in M\left(n \times\left(m-1-m^{*}\right)\right)$ such that for all $t \in[\bar{t}-\eta, \bar{t}+\eta]$

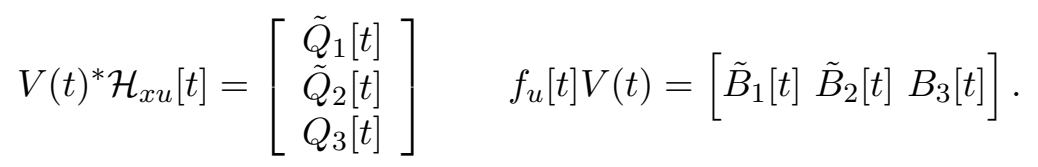


Thus $\tilde{Q}_{1}[t] \tilde{B}_{1}[t]=\left(\tilde{Q}_{1}[t] \tilde{B}_{1}[t]\right)^{*}$ for all $t \in \Upsilon$ and if $m^{*}<m-1$, then

$$
\tilde{Q}_{1}[\tau] \tilde{B}_{2}[\tau] \neq\left(\tilde{Q}_{2}[\tau] \tilde{B}_{1}[\tau]\right)^{*} .
$$

Fix $\tilde{u} \in \mathbb{R}^{m-1} \times\{0\}, \tilde{u} \neq 0$ and let $\tilde{u}^{1} \in \mathbb{R}^{m^{*}}, \tilde{u}^{2} \in \mathbb{R}^{m-1-m^{*}}$ be such that $\tilde{u}=\left(\tilde{u}^{1}, \tilde{u}^{2}, 0\right)$. If $m^{*}=m-1$, then $\tilde{u}^{2}$ is absent in this decomposition. For any small $\varepsilon>0$ define

$$
\tilde{u}_{\varepsilon}^{1}(t):=\left\{\begin{array}{ll}
\frac{1}{\left|E_{\varepsilon}^{1}\right|} \tilde{u}^{1} & t \in E_{\varepsilon}^{1} \\
-\frac{1}{\left|E_{\varepsilon}^{1}\right|} \tilde{u}^{1} & t \in E_{\varepsilon}^{2} \\
0 & \text { otherwise }
\end{array} \quad \tilde{u}_{\varepsilon}^{2}(t):=\left\{\begin{array}{ll}
\tilde{u}^{2} & t \in E_{\varepsilon}^{1} \cup E_{\varepsilon}^{2} \\
0 & \text { otherwise }
\end{array}, \quad \tilde{u}_{\varepsilon}^{3}(t):=0 .\right.\right.
$$

With this definition $V(t) \tilde{u}_{\varepsilon}(t) \in S(t)$ for all $t \in \Upsilon \cap E_{\varepsilon}$, where $\tilde{u}_{\varepsilon}(t)=\left(\tilde{u}_{\varepsilon}^{1}(t), \tilde{u}_{\varepsilon}^{2}(t), 0\right)$. Hence, $V(\cdot) \tilde{u}_{\varepsilon}(\cdot) \in \mathcal{U}_{A}$. By Remark 5.4 and assumption (i), Theorem 5.3 implies that

$$
-\frac{1}{2} y_{\varepsilon}(1)^{*} \varphi^{\prime \prime}(\bar{x}(1)) y_{\varepsilon}(1)+\frac{1}{2} \int_{0}^{1} y_{\varepsilon}(t)^{*} \mathcal{H}_{x x}[t] y_{\varepsilon}(t) d t+\int_{\tau-\delta_{\varepsilon}}^{\tau+\delta_{\varepsilon}} \tilde{u}_{\varepsilon}(t)^{*} V(t)^{*} \mathcal{H}_{x u}[t] y_{\varepsilon}(t) d t \leq 0
$$

where $y_{\varepsilon}(\cdot)$ is the solution of (2.1) for $u(\cdot)=V(\cdot) \tilde{u}_{\varepsilon}(\cdot)$. Define for all $t \in[0,1]$

$$
\tilde{\zeta}_{\varepsilon}(t):=\int_{0}^{t} \tilde{u}_{\varepsilon}(s) d s .
$$

Then, $\left\|\tilde{\zeta}_{\varepsilon}^{1}(\cdot)\right\|_{\infty} \leq\left\|\tilde{u}_{1}\right\|$ and, as in $(3.4), \int_{0}^{1}\left\|\tilde{\zeta}_{\varepsilon}^{1}(t)\right\| d t=O\left(\left|\Upsilon \cap E_{\varepsilon}\right|\right), \tilde{\zeta}_{\varepsilon}^{1}(t)=0$ outside of the interval $\left(\tau-\delta_{\varepsilon}, \tau+\delta_{\varepsilon}\right)$. To simplify the notation we will omit for a while the subscript $\varepsilon$.

Performing integrations by parts as in the proof of Theorem 3.1, from (5.4) we obtain

$$
\begin{aligned}
& -\frac{1}{2} y(1)^{*} \varphi^{\prime \prime}(\bar{x}(1)) y(1)+\left.\tilde{\zeta}(\cdot)^{*} V(\cdot)^{*} \mathcal{H}_{x u}[\cdot] y(\cdot)\right|_{\tau-\delta} ^{\tau+\delta}+\frac{1}{2} \int_{0}^{1} y(t)^{*} \mathcal{H}_{x x}[t] y(t) d t \\
& -\int_{\tau-\delta}^{\tau+\delta} \tilde{\zeta}(t)^{*} \frac{d}{d t}\left(V^{*} \mathcal{H}_{x u}\right)[t] y(t) d t-\int_{\tau-\delta}^{\tau+\delta} \tilde{\zeta}(t)^{*} V(t)^{*} \mathcal{H}_{x u}[t] f_{x}[t] y(t) d t \\
& +\frac{1}{2} \int_{\tau-\delta}^{\tau+\delta} \tilde{\zeta}^{1}(t)^{*} \frac{d}{d t}\left(\tilde{Q}_{1} \tilde{B}_{1}\right)[t] \tilde{\zeta}^{1}(t) d t-\int_{\tau-\delta}^{\tau+\delta} \tilde{\zeta}^{2}(t)^{*} \tilde{Q}_{2}[t] \tilde{B}_{2}[t] \tilde{u}^{2}(t) d t \\
& -\int_{\tau-\delta}^{\tau+\delta} \tilde{\zeta}^{1}(t)^{*}\left(\tilde{Q}_{1}[t] \tilde{B}_{2}[t]-\left(\tilde{Q}_{2}[t] \tilde{B}_{1}[t]\right)^{*}\right) \tilde{u}^{2}(t) d t+\int_{\tau-\delta}^{\tau+\delta} \tilde{\zeta}^{2}(t)^{*} \frac{d}{d t}\left(\tilde{Q}_{2} \tilde{B}_{1}\right)[t] \tilde{\zeta}^{1}(t) d t \leq 0 .
\end{aligned}
$$

For all $t \in[0,1]$, define

$$
\xi(t):=X(t) \int_{0}^{t} X^{-1}(s)\left(f_{x}[s] \tilde{B}_{1}[s]-\frac{d}{d s} \tilde{B}_{1}[s]\right) \tilde{\zeta}^{1}(s) d s+X(t) \int_{0}^{t} X^{-1}(s) \tilde{B}_{2}[s] \tilde{u}^{2}(s) d s
$$

and observe that, as in the proof of Theorem $3.1, y(\cdot)=\xi(\cdot)+\tilde{B}_{1}[\cdot] \tilde{\zeta}^{1}(\cdot)$.

We restore the subscript $\varepsilon$. Thanks to the definition of $\tilde{u}_{\varepsilon}(\cdot),\left\|\xi_{\varepsilon}(\cdot)\right\|_{\infty}=O\left(\left|\Upsilon \cap E_{\varepsilon}\right|\right)$ and

$$
\begin{gathered}
\xi_{\varepsilon}(1)^{*} \varphi^{\prime \prime}(\bar{x}(1)) \xi_{\varepsilon}(1)=o\left(\left|\Upsilon \cap E_{\varepsilon}\right|\right), \quad \int_{0}^{1} \xi_{\varepsilon}(t)^{*} \mathcal{H}_{x x}[t] \xi_{\varepsilon}(t) d t=o\left(\left|\Upsilon \cap E_{\varepsilon}\right|\right), \\
\int_{\tau-\delta_{\varepsilon}}^{\tau+\delta_{\varepsilon}} \tilde{\zeta}_{\varepsilon}^{1}(t)^{*} \tilde{B}_{1}[t]^{*} \mathcal{H}_{x x}[t] \xi_{\varepsilon}(t) d t=o\left(\left|\Upsilon \cap E_{\varepsilon}\right|\right), \quad \int_{\tau-\delta_{\varepsilon}}^{\tau+\delta_{\varepsilon}} \tilde{\zeta}_{\varepsilon}^{2}(t)^{*} \tilde{Q}_{2}[t] \tilde{B}_{2}[t] \tilde{u}_{\varepsilon}^{2}(t)=o\left(\left|\Upsilon \cap E_{\varepsilon}\right|\right), \\
\int_{\tau-\delta_{\varepsilon}}^{\tau+\delta_{\varepsilon}} \tilde{\zeta}_{\varepsilon}(t)^{*}\left(\frac{d}{d t}\left(V^{*} \mathcal{H}_{x u}\right)[t]+V(t)^{*} \mathcal{H}_{x u}[t] f_{x}[t]\right) \xi_{\varepsilon}(t) d t=o\left(\left|\Upsilon \cap E_{\varepsilon}\right|\right), \\
\int_{\tau-\delta_{\varepsilon}}^{\tau+\delta_{\varepsilon}} \tilde{\zeta}_{\varepsilon}^{2}(t)^{*}\left(-\frac{d}{d t} \tilde{Q}_{2}[t] \tilde{B}_{1}[t]-\tilde{Q}_{2}[t] f_{x}[t] \tilde{B}_{1}[t]+\frac{d}{d t}\left(\tilde{Q}_{2} \tilde{B}_{1}\right)[t]\right) \tilde{\zeta}_{\varepsilon}^{1}(t) d t=o\left(\left|\Upsilon \cap E_{\varepsilon}\right|\right), \\
\left.\tilde{\zeta}_{\varepsilon}(\cdot)^{*} V(\cdot)^{*} \mathcal{H}_{x u}[\cdot] \xi_{\varepsilon}(\cdot)\right|_{\tau-\delta_{\varepsilon}} ^{\tau+\delta_{\varepsilon}}=o\left(\left|\Upsilon \cap E_{\varepsilon}\right|\right) .
\end{gathered}
$$


Substituting $y_{\varepsilon}(\cdot)$ with $\xi_{\varepsilon}(\cdot)+\tilde{B}_{1}[\cdot] \tilde{\zeta}_{\varepsilon}^{1}(\cdot)$ in 5.5 and using the above estimates, similarly to the proof of Theorem 3.1 , we get

$$
\begin{aligned}
& \int_{\tau-\delta_{\varepsilon}}^{\tau+\delta_{\varepsilon}} \tilde{\zeta}_{\varepsilon}^{1}(t)^{*}\left(\frac{1}{2} \tilde{B}_{1}[t]^{*} \mathcal{H}_{x x}[t] \tilde{B}_{1}[t]-\frac{d}{d t} \tilde{Q}_{1}[t] \tilde{B}_{1}[t]-\tilde{Q}_{1}[t] f_{x}[t] \tilde{B}_{1}[t]\right) \tilde{\zeta}_{\varepsilon}^{1}(t) d t \\
& +\frac{1}{2} \int_{\tau-\delta_{\varepsilon}}^{\tau+\delta_{\varepsilon}} \tilde{\zeta}_{\varepsilon}^{1}(t)^{*} \frac{d}{d t}\left(\tilde{Q}_{1} \tilde{B}_{1}\right)[t] \tilde{\zeta}_{\varepsilon}^{1}(t) d t \\
& -\int_{\tau-\delta_{\varepsilon}}^{\tau+\delta_{\varepsilon}} \tilde{\zeta}_{\varepsilon}^{1}(t)^{*}\left(\tilde{Q}_{1}[t] \tilde{B}_{2}[t]-\left(\tilde{Q}_{2}[t] \tilde{B}_{1}[t]\right)^{*}\right) \tilde{u}_{\varepsilon}^{2}(t) d t \leq o\left(\left|\Upsilon \cap E_{\varepsilon}\right|\right) .
\end{aligned}
$$

Therefore, applying Lemma 2.6, dividing by $\left|\Upsilon \cap E_{\varepsilon}\right|$ in $(5.6)$ and passing to the limit when $\varepsilon \rightarrow 0+$, we obtain

$$
\begin{aligned}
& \frac{1}{3}\left(\tilde{u}^{1}\right)^{*}\left(\frac{1}{2} \tilde{B}_{1}[\tau]^{*} \mathcal{H}_{x x}[\tau] \tilde{B}_{1}[\tau]-\frac{1}{2} \frac{d}{d t} \tilde{Q}_{1}[\tau] \tilde{B}_{1}[\tau]-\tilde{Q}_{1}[\tau] f_{x}[\tau] \tilde{B}_{1}[\tau]+\frac{1}{2} \tilde{Q}_{1}[\tau] \frac{d}{d t} \tilde{B}_{1}[\tau]\right) \tilde{u}^{1} \\
& -\frac{1}{2}\left(\tilde{u}^{1}\right)^{*}\left(\tilde{Q}_{1}[\tau] \tilde{B}_{2}[\tau]-\left(\tilde{Q}_{2}[\tau] \tilde{B}_{1}[\tau]\right)^{*}\right) \tilde{u}^{2} \leq 0 .
\end{aligned}
$$

By the arbitrariness of $\left(\left(\tilde{u}^{1}\right)^{*},\left(\tilde{u}^{2}\right)^{*}\right)^{*} \in \mathbb{R}^{m-1}$, applying the same arguments as at the end of the proof of Theorem 3.1 , we deduce that if $m^{*}<m-1$, then $\tilde{Q}_{1}[\tau] \tilde{B}_{2}[\tau]-\left(\tilde{Q}_{2}[\tau] \tilde{B}_{1}[\tau]\right)^{*}=0$ contradicting (5.3). Thus $m^{*}=m-1$. From (5.7) it follows that

$$
\frac{1}{2} B_{1}[\tau]^{*} \mathcal{H}_{x x}[\tau] B_{1}[\tau]-\frac{1}{2} \frac{d}{d t} Q_{1}[\tau] B_{1}[\tau]-Q_{1}[\tau] f_{x}[\tau] B_{1}[\tau]+\frac{1}{2} Q_{1}[\tau] \frac{d}{d t} B_{1}[\tau]
$$

is negative semi-definite on $\mathbb{R}^{m-1}$.

Theorem 5.7. Let $(\bar{x}(\cdot), \bar{u}(\cdot))$ be a strong local minimizer for the Mayer problem (1.3) and $p(\cdot)$ be as in the maximum principle of Theorem 2.3. Assume that for some $0 \leq t_{1}<t_{2} \leq 1, \bar{u}(\cdot)$ is Lipschitz on $\left[t_{1}, t_{2}\right], b_{U}(\cdot) \in C^{2}$ on a neighborhood of $\bar{u}\left(\left[t_{1}, t_{2}\right]\right)$ and (5.2) holds true. Then for a.e. $t \in\left(t_{1}, t_{2}\right), \mathcal{H}_{x u}[t] f_{u}[t]$ is symmetric on the subspace $P_{\bar{u}(t)}$ and

$$
\begin{gathered}
\frac{1}{2} f_{u}[t]^{*} \mathcal{H}_{x x}[t] f_{u}[t]-\frac{1}{2} \frac{d}{d t} \mathcal{H}_{x u}[t] f_{u}[t]-\mathcal{H}_{x u}[t] f_{x}[t] f_{u}[t]+\frac{1}{2} \mathcal{H}_{x u}[t] \frac{d}{d t} f_{u}[t] \\
-\frac{1}{2}\left(V(t)^{-1}\right)^{*} V^{\prime}(t)^{*} \mathcal{H}_{x u}[t] f_{u}[t]+\frac{1}{2} \mathcal{H}_{x u}[t] f_{u}[t] V^{\prime}(t) V(t)^{-1}
\end{gathered}
$$

is negative semi-definite on $P_{\bar{u}(t)}$, where $V$ is as defined just before Lemma 5.6.

Proof. By Theorem 3.1 the conclusions hold true for a.e. $t \in\left[t_{1}, t_{2}\right]$ such that $\bar{u}(t) \in \operatorname{Int} U$. Lemma 5.6 implies that they are also verified for a.e. $t \in\left(t_{1}, t_{2}\right)$ such that $\bar{u}(t) \in \partial U$.

Note that the above theorem brings a new information for a.e. $t \in\left(t_{1}, t_{2}\right)$ such that $\bar{u}(t) \in \partial U$. When $\bar{u}(t) \in$ Int $U$, stronger results are provided in Section 3 .

\section{MAYER'S PROBlem With ENDPOINT CONSTRAintS}

Let $K$ be a proper closed nonempty subset of $\mathbb{R}^{n}$ of class $C^{2}$. Then the oriented distance $b_{K}(\cdot)$ is $C^{2}$ on a neighborhood of $\partial K$, cf. [11]. Denote by $S_{[0,1]}^{K}\left(x_{0}\right)$ the set of all $x(\cdot) \in S_{[0,1]}\left(x_{0}\right)$ satisfying $x(1) \in K$. We call such trajectories feasible. We study here the following Mayer optimal control problem

$$
\min \left\{\varphi(x(1)): x(\cdot) \in S_{[0,1]}^{K}\left(x_{0}\right)\right\} .
$$

Let $(\bar{x}(\cdot), \bar{u}(\cdot))$ be a strong local minimizer of the above problem and $P_{\bar{u}(t)}$ be defined as in Section 2. If $\bar{x}(1)$ belongs to Int $K$, then the previously developed second order variational analysis does apply as though there were no endpoint constraints. This is due to the fact that 
small perturbations of $\bar{u}(\cdot)$ lead to feasible trajectories. For this reason we have to investigate only the case $\bar{x}(1) \in \partial K$. Since the boundary of $K$ is smooth, the limiting normal cone to $K$ at $\bar{x}(1)$ is equal to $\mathbb{R}_{+} \nabla b_{K}(\bar{x}(1))$. Thus we deduce, for instance from [23], the following maximum principle.

Theorem 6.1. Let $(\bar{x}(\cdot), \bar{u}(\cdot))$ be a strong local minimizer for 6.1) with $\bar{x}(1) \in \partial K$. Then there exists an absolutely continuous function $p:[0,1] \rightarrow \mathbb{R}^{n}$ and $\lambda \in\{0,1\}, \mu \geq 0$, satisfying $\lambda+\mu>0,(1.4$, 1.5) and the transversality condition

$$
-p(1)=\lambda \nabla \varphi(\bar{x}(1))+\mu \nabla b_{K}(\bar{x}(1)) .
$$

The above maximum principle may be abnormal, that is $\lambda=0$. In this case $p(1)$ may be taken equal to $-\nabla b_{K}(\bar{x}(1))$.

Lemma 6.2. Let $(\bar{x}(\cdot), \bar{u}(\cdot))$ be a strong local minimizer for 6.1 with $\bar{x}(1) \in \partial K$ for which an abnormal maximum principle of Theorem 6.1 holds true for some $\mu>0$. Then for every measurable bounded selection $u(t) \in P_{\bar{u}(t)}$ a.e. in $[0,1]$, the solution $y(\cdot)$ of (2.1) satisfies $\left\langle\nabla b_{K}(\bar{x}(1)), y(1)\right\rangle=0$ and $\left\langle p(t), f_{u}[t] u(t)\right\rangle=0$ a.e. in $[0,1]$.

Proof. Let $u:[0,1] \rightarrow \mathbb{R}^{m}$ be as above. By $(1.5), \mathcal{H}_{u}[t] u(t)=\left\langle p(t), f_{u}[t] u(t)\right\rangle=0$ a.e. in $[0,1]$. Then from (1.4) and (2.1) we obtain

$$
\langle p(1), y(1)\rangle=\int_{0}^{1}\langle p, y\rangle^{\prime}(t) d t=\int_{0}^{1}\left(\left\langle p^{\prime}(t), y(t)\right\rangle+\left\langle p(t), y^{\prime}(t)\right\rangle\right) d t=\int_{0}^{1} \mathcal{H}_{u}[t] u(t)=0 .
$$

If the maximum principle is abnormal, then the cost is not involved into the first order optimality conditions. Thus inequality (2.3) can not be related to the costate $p(\cdot)$ as it was done in the proof of Theorem 2.5. To apply the variational approach developed in the previous sections, we use the exact penalization which allows to deal with a normal maximum principle.

Definition 6.3. The Mayer problem (6.1) is called calm at $\bar{x}(\cdot)$ if exist $\varepsilon>0$ and $M>0$ such that for every $\nu \in(0, \varepsilon)$ and $x(\cdot) \in S_{[0,1]}\left(x_{0}\right)$ satisfying $d_{K}(x(1)) \leq \nu$ and $\|x(\cdot)-\bar{x}(\cdot)\|_{\infty} \leq \varepsilon$ we have $\varphi(x(1)) \geq \varphi(\bar{x}(1))-M \nu$.

Assuming that the Mayer problem (6.1) is calm at $\bar{x}(\cdot)$ we can apply Proposition 6.4.3 from [10] to deduce that there exists $k>0$ such that $(\bar{x}(\cdot), \bar{u}(\cdot))$ is a strong local minimizer of the following minimization problem without endpoint constraints:

$$
\min \left\{\varphi(x(1))+k d_{K}(x(1)): x(\cdot) \in S_{[0,1]}\left(x_{0}\right)\right\} .
$$

Since $\varphi(\cdot)+k d_{K}(\cdot)$ is no longer $C^{2}$, we need the following (non smooth) maximum principle that can be deduced, for instance, from [23].

Theorem 6.4. Let $(\bar{x}(\cdot), \bar{u}(\cdot))$ be a strong local minimizer of 6.2 with $\bar{x}(1) \in \partial K$. Then there exists an absolutely continuous function $p:[0,1] \rightarrow \mathbb{R}^{n}$ satisfying (1.4), (1.5) and the transversality condition

$$
-p(1) \in \nabla \varphi(\bar{x}(1))+k\left[0, \nabla b_{K}(\bar{x}(1))\right],
$$

where $\left[0, \nabla b_{K}(\bar{x}(1))\right]=\left\{\mu \nabla b_{K}(\bar{x}(1)): \mu \in[0,1]\right\}$.

Denote by $q^{1}(\cdot)$ the solution of 1.4 for $p(1)=-\nabla \varphi(\bar{x}(1))$ and by $q^{2}(\cdot)$ the solution of (1.4) for $p(1)=-\nabla \varphi(\bar{x}(1))-k \nabla b_{K}\left(\bar{x}(1)\right.$. Note that $q^{i}(1)$ are extremal points in the transversality condition (6.3). The mappings $q^{i}(\cdot)$ do not satisfy, in general, the maximum principle (1.5) but are used below to express the second order necessary optimality conditions. Define for $i=1,2$

$$
\varphi^{1}(\cdot):=\varphi(\cdot), \varphi^{2}(\cdot):=\varphi(\cdot)+k b_{K}(\cdot), \quad \mathcal{H}_{x x}^{i}[\cdot]:=\mathcal{H}_{x x}\left(\cdot, \bar{x}(\cdot), q^{i}(\cdot), \bar{u}(\cdot)\right) .
$$

The mappings $\mathcal{H}_{x u}^{i}[\cdot]$ and $\mathcal{H}_{u u}^{i}[\cdot]$ are defined in a similar way. 
Theorem 6.5. Let $(\bar{x}(\cdot), \bar{u}(\cdot))$ be a strong local minimizer for 6.2) with $\bar{x}(1) \in \partial K$ and $p(\cdot)$ be as in Theorem 6.4. Then for every $u(\cdot) \in \mathcal{U}_{A}$ such that $\mathcal{H}_{u}[\cdot] u(\cdot)=0$ and the corresponding solution $y(\cdot)$ of (2.1) verifies $\left\langle\nabla b_{K}(\bar{x}(1)), y(1)\right\rangle=0$, and for any $v(\cdot) \in L^{\infty}\left([0,1] ; \mathbb{R}^{m}\right)$ satisfying $v(t) \in T_{U}^{b(2)}(\bar{u}(t), u(t))$ for a.e. $t \in A$, there exists $i \in\{1,2\}$ such that

$$
\begin{aligned}
0 \geq & \int_{0}^{1}\left(\mathcal{H}_{u}^{i}[t] v(t)+\frac{1}{2} y(t)^{*} \mathcal{H}_{x x}^{i}[t] y(t)+u(t)^{*} \mathcal{H}_{x u}^{i}[t] y(t)+\frac{1}{2} u(t)^{*} \mathcal{H}_{u u}^{i}[t] u(t)\right) d t \\
& -\frac{1}{2} y(1)^{*}\left(\varphi^{i}\right)^{\prime \prime}(\bar{x}(1)) y(1) .
\end{aligned}
$$

Proof. Let $u(\cdot), v(\cdot), y(\cdot)$ be as in the statement of the theorem and $\tilde{x}_{h}(\cdot), w(\cdot)$ be as in the proof of Theorem 2.5. Then for all small $h>0$,

$$
0 \geq \varphi(\bar{x}(1))-\varphi\left(\tilde{x}_{h}(1)\right)-k d_{K}\left(\tilde{x}_{h}(1)\right) .
$$

By the transversality condition for some $\mu \in[0,1],-p(1)=\nabla \varphi(\bar{x}(1))+k \mu \nabla b_{K}(\bar{x}(1))$ and by the same arguments as in the proof of Lemma 6.2 , $\langle p(1), y(1)\rangle=0$.

Suppose first that there exists a sequence $h_{i} \rightarrow 0+$ such that $\tilde{x}_{h_{i}}(1) \notin K$. Then, by (6.5),

$$
\begin{aligned}
0 \geq & \varphi(\bar{x}(1))-\varphi\left(\tilde{x}_{h_{i}}(1)\right)-k b_{K}\left(\tilde{x}_{h_{i}}(1)\right)=-\left\langle\nabla \varphi(\bar{x}(1))+k \nabla b_{K}(\bar{x}(1)), h_{i} y(1)+h_{i}^{2} w(1)\right\rangle \\
& -\frac{h_{i}^{2}}{2} y(1)^{*}\left(\varphi^{\prime \prime}(\bar{x}(1))+k b_{K}^{\prime \prime}(\bar{x}(1))\right) y(1)+o\left(h_{i}^{2}\right) .
\end{aligned}
$$

Since $\left\langle\nabla b_{K}(\bar{x}(1)), y(1)\right\rangle=0$, we deduce from $\langle p(1), y(1)\rangle=0$ that $\langle\nabla \varphi(\bar{x}(1)), y(1)\rangle=0$ and therefore $\left\langle\nabla \varphi(\bar{x}(1))+k \nabla b_{K}(\bar{x}(1)), y(1)\right\rangle=0$. From $(6.6)$, dividing by $h_{i}^{2}$ and passing to the limit we obtain

$$
0 \geq-\left\langle\nabla \varphi(\bar{x}(1))+k \nabla b_{K}(\bar{x}(1)), w(1)\right\rangle-\frac{1}{2} y(1)^{*}\left(\varphi^{\prime \prime}(\bar{x}(1))+k b_{K}^{\prime \prime}(\bar{x}(1))\right) y(1) .
$$

In the same way as in the proof of Theorem 2.5 , the last inequality and $(2.2)$ imply (6.4) for $i=2$.

Suppose next that for all small $h>0, \tilde{x}_{h}(1) \in K$. Then 6.5 yields

$$
0 \geq-\left\langle\nabla \varphi(\bar{x}(1)), h y(1)+h^{2} w(1)\right\rangle-\frac{h^{2}}{2} y(1)^{*} \varphi^{\prime \prime}(\bar{x}(1)) y(1)+o\left(h^{2}\right) .
$$

Since $\left\langle\nabla b_{K}(\bar{x}(1), y(1)\rangle=0\right.$,

$$
-\langle\nabla \varphi(\bar{x}(1)), y(1)\rangle=-\left\langle\nabla \varphi(\bar{x}(1))+k \mu \nabla b_{K}(\bar{x}(1)), y(1)\right\rangle=\langle p(1), y(1)\rangle=0 .
$$

Hence, dividing (6.7) by $h^{2}$ and letting $h \rightarrow 0$ we get

$$
0 \geq-\langle\nabla \varphi(\bar{x}(1)), w(1)\rangle-\frac{1}{2} y(1)^{*} \varphi^{\prime \prime}(\bar{x}(1)) y(1) .
$$

Using (2.2), similarly to the proof of Theorem 2.5, we deduce (6.4) from (6.8) for $i=1$.

Proposition 6.6. Let $(\bar{x}(\cdot), \bar{u}(\cdot))$ be a strong local minimizer for 6.1) with $\bar{x}(1) \in \partial K$. Consider $u(\cdot) \in \mathcal{U}_{A}$ and the corresponding solution $y(\cdot)$ of 2.1 . If $\left\langle\nabla b_{K}(\overline{\bar{x}}(1)), y(1)\right\rangle<0$, then

$$
\langle\nabla \varphi(\bar{x}(1)), y(1)\rangle \geq 0 .
$$

Let $p(\cdot)$ be as in the maximum principle of Theorem 6.1. If $\langle\nabla \varphi(\bar{x}(1)), y(1)\rangle=0, \mathcal{H}_{u}[\cdot] u(\cdot)=$ 0 , then $p(1)=-\nabla \varphi(\bar{x}(1))$ and for any $v(\cdot) \in L^{\infty}\left([0,1] ; \mathbb{R}^{m}\right)$ satisfying $v(t) \in T_{U}^{b(2)}(\bar{u}(t), u(t))$ for a.e. $t \in A$, we have

$$
\langle\nabla \varphi(\bar{x}(1)), w(1)\rangle+\frac{1}{2} y(1)^{*} \varphi^{\prime \prime}(\bar{x}(1)) y(1) \geq 0,
$$

where $w(\cdot)$ solves (2.2). 
Proof. By [18] there exist measurable controls $u_{h}(\cdot)$ such that for the corresponding solutions $x_{h}(\cdot)$ of 1.2 the difference quotients $\frac{x_{h}-\bar{x}}{h}$ converge uniformly to $y(\cdot)$ when $h \rightarrow 0+$. Furthermore, from the inequality $\left\langle\nabla b_{K}(\bar{x}(1)), y(1)\right\rangle<0$, we deduce that $x_{h}(1) \in K$ for all small $h>0$. Hence $\varphi\left(x_{h}(1)\right) \geq \varphi(\bar{x}(1))$ and therefore $\langle\nabla \varphi(\bar{x}(1)), y(1)\rangle \geq 0$.

Let $p(\cdot), v(\cdot)$ be as in the second statement of the proposition, $\mu \geq 0$ be as in the maximum principle of Theorem 6.1 and $\tilde{x}_{h}(\cdot)$ be as in the proof of Theorem 2.5. Then it is not difficult to realize that it is feasible whenever $h>0$ is small enough. If $\mathcal{H}_{u}[t] u(t)=0$ a.e. in $[0,1]$, then, by the proof of Lemma 6.2. $\langle p(1), y(1)\rangle=0$. If $\langle\nabla \varphi(\bar{x}(1)), y(1)\rangle=0$, then the transversality condition of the maximum principle yields $\mu\left\langle\nabla b_{K}(\bar{x}(1)), y(1)\right\rangle=0$. Consequently, $\mu=0$. The proofs ends by using the inequality (2.3).

The above proposition provides a first and a second order necessary optimality conditions only in the case when $\left\langle\nabla b_{K}(\bar{x}(1)), y(1)\right\rangle<0$. Observe that if $\left\langle\nabla b_{K}(\bar{x}(1)), y(1)\right\rangle>0$, then $x_{h}(\cdot), \tilde{x}_{h}(\cdot)$ from the proof of our proposition are not feasible for all small $h>0$ and so this case may be ruled out in our analysis.

Below we provide second order necessary optimality conditions obtained by analyzing some particular $u(\cdot) \in \mathcal{U}_{A}$ with $u(t) \in P_{\bar{u}(t)}$ a.e. for which the corresponding solutions $y(\cdot)$ of (2.1) satisfy $\left\langle\nabla b_{K}(\bar{x}(1)), y(1)\right\rangle=0$.

Consider the solution $q(\cdot)$ of the adjoint system (1.4) such that $q(1)=-\nabla b_{K}(\bar{x}(1))$ and define the subspaces $\mathcal{T}_{t} \subset P_{\bar{u}(t)}$ by

$$
\mathcal{T}_{t}:=\left\{u \in P_{\bar{u}(t)}:\left\langle q(t), f_{u}[t] u\right\rangle=0\right\} .
$$

Notice that, by Lemma 6.2, if the abnormal maximum principle of Theorem 6.1 holds true, then $\mathcal{T}_{t}=P_{\bar{u}(t)}$ a.e. in $[0,1]$. Observe next that for every measurable essentially bounded selection $u(t) \in \mathcal{T}_{t}$ and the corresponding solution $y(\cdot)$ of $(2.1)$ we have

$$
\left\langle\nabla b_{K}(\bar{x}(1)), y(1)\right\rangle=-\langle q(1), y(1)\rangle=-\int_{0}^{1}\langle q, y\rangle^{\prime}(t) d t=-\int_{0}^{1}\left\langle q(t), f_{u}[t] u(t)\right\rangle d t=0 .
$$

Therefore, by Theorem 6.5. (6.4) holds true for every $u(\cdot) \in \mathcal{U}_{A}$ such that $u(t) \in \mathcal{T}_{t}$ a.e. in $[0,1]$. This and the same arguments as those from Sections 3, 4, 5 allow to deduce from (6.4) the following three theorems.

Theorem 6.7. Let $(\bar{x}(\cdot), \bar{u}(\cdot))$ be a strong local minimizer of the Mayer problem (6.1) such that $\bar{x}(1) \in \partial K$. Assume that (6.1) is calm at $\bar{x}(\cdot)$ and for some $0 \leq t_{1}<t_{2} \leq 1, \bar{u}(\cdot)$ is integrable on $\left[t_{1}, t_{2}\right]$ and for $j=1,2$

$$
\left\{\begin{aligned}
(i) & \mathcal{H}_{u u}^{j}[t]=0 \text { a.e. in }\left[t_{1}, t_{2}\right] \\
\text { (ii) } & \mathcal{H}_{x u}^{j}[\cdot], f_{u}[\cdot] \text { are Lipschitz on }\left[t_{1}, t_{2}\right] ; \\
\text { (iii) } & \mathcal{H}_{x x}^{j}[\cdot], f_{x}[\cdot] \text { are essentially bounded on }\left[t_{1}, t_{2}\right] .
\end{aligned}\right.
$$

Then for a.e. $t \in\left[t_{1}, t_{2}\right]$ with $\bar{u}(t) \in \operatorname{Int} U$ there exists $i \in\{1,2\}$ such that $\mathcal{H}_{x u}^{i}[t] f_{u}[t]$ is symmetric on $\mathcal{T}_{t}$ and

$$
R^{i}(t):=\frac{1}{2} f_{u}[t]^{*} \mathcal{H}_{x x}^{i}[t] f_{u}[t]-\frac{1}{2} \frac{d}{d t} \mathcal{H}_{x u}^{i}[t] f_{u}[t]-\mathcal{H}_{x u}^{i}[t] f_{x}[t] f_{u}[t]+\frac{1}{2} \mathcal{H}_{x u}^{i}[t] \frac{d}{d t} f_{u}[t]
$$

is negative semi-definite on $\mathcal{T}_{t}$.

Theorem 6.8. Let $U$ be a convex polytope and $(\bar{x}(\cdot), \bar{u}(\cdot))$ be a strong local minimizer of the Mayer problem (6.1) such that $\bar{x}(1) \in \partial K$. Assume that (6.1) is calm at $\bar{x}(\cdot)$ and for some $0 \leq t_{1}<t_{2} \leq 1, \bar{u}(\cdot)$ is integrable on $\left[t_{1}, t_{2}\right]$ and (6.9) holds true for $j=1,2$. Then for a.e. $t \in\left[t_{1}, t_{2}\right]$ there exists $i \in\{1,2\}$ such that $\mathcal{H}_{x u}^{i}[t] f_{u}[t]$ is symmetric on $\mathcal{T}_{t}$ and $R^{i}(t)$ is negative semi-definite on $\mathcal{T}_{t}$. 
Corollary 6.9. Under all the assumptions of Theorem 6.8 suppose that $f$ is as in (1.10), where $f_{l}(\cdot) \in C^{2}\left(\mathbb{R}^{n} ; \mathbb{R}^{n}\right)$ and $U_{l} \subset \mathbb{R}$ is a closed (possibly unbounded) interval for all $l \in\{1, \ldots, m\}$. For $i=1,2$ define the matrix valued mappings $\Theta^{i}:[0,1] \rightarrow M(m \times m)$, where

$$
\theta_{k, l}^{i}(t):=\left\langle q^{i}(t),\left[f_{k}, f_{l}\right](\bar{x}(t))\right\rangle \quad \forall k, l \in\{1, \ldots, m\} .
$$

Then for a.e. $t \in\left[t_{1}, t_{2}\right]$ there exists $i \in\{1,2\}$ such that $\Theta^{i}(t)\left(\mathcal{T}_{t}\right)$ is orthogonal to $\mathcal{T}_{t}$.

In the next theorem subspaces $S(s)$ are defined as in Section 5 .

Theorem 6.10. Let $U$ be of class $C^{2}$ and $(\bar{x}(\cdot), \bar{u}(\cdot))$ be a strong local minimizer of the Mayer problem (6.1) such that $\bar{x}(1) \in \partial K$. Assume that (6.1) is calm at $\bar{x}(\cdot)$ and for some $0 \leq t_{1}<$ $t_{2} \leq 1, \bar{u}(\cdot)$ is integrable on $\left[t_{1}, t_{2}\right]$. Then for a.e. $t \in\left(t_{1}, t_{2}\right)$ such that $\bar{u}(t) \in \partial U$ and for $j=1,2$ and some $\delta>0$

$$
\left\{\begin{array}{l}
(a) \quad \bar{u}(\cdot), \mathcal{H}_{x u}^{j}[\cdot], f_{u}[\cdot] \text { are Lipschitz on }(t-\delta, t+\delta) \\
\text { (b) } u^{*}\left(\mathcal{H}_{u u}^{j}[s]-\left\|\mathcal{H}_{u}^{j}[s]\right\| b_{U}^{\prime \prime}(\bar{u}(s))\right) u=0 \forall u \in S(s) \text { and a.e. } s \in(t-\delta, t+\delta) ; \\
\text { (c) } \mathcal{H}_{x x}^{j}[\cdot], f_{x}[\cdot] \text { are essentially bounded on }(t-\delta, t+\delta)
\end{array}\right.
$$

there exists $i \in\{1,2\}$ for which $\mathcal{H}_{x u}^{i}[t] f_{u}[t]$ is symmetric on $\mathcal{T}_{t}$.

Remark 6.11. Similar analysis can be performed when $K$ is an intersection of a finite family of closed sets $K_{j}$ of class $C^{2}$. Then the assumptions and statements will involve Hamiltonians $\mathcal{H}^{j}$ for each active index $j$.

Acknowledgement. This work was co-funded by the European Union under the 7th Framework Programme "FP7-PEOPLE-2010-ITN", grant agreement number 264735-SADCO.

\section{REFERENCES}

[1] A.A. Agračev and R.V. Gamkrelidze. The principle of second order optimality for time-optimal problems. Mat. Sb. (N.S.), 100(142)(4):610-643, 648, 1976.

[2] A.A. Agrachev. A second order necessary condition for optimality in the general nonlinear case. Math. USSR Sbornik, 31:493-506, 1977.

[3] A.A. Agrachev and Y.L. Sachkov. Control theory from the geometric viewpoint, volume 87 of Encyclopaedia of Mathematical Sciences. Springer-Verlag, Berlin, 2004.

[4] A.A. Agrachev and A. V. Sarychev. Abnormal sub-riemannian geodesics: Morse index and rigidity. Annales de l'IHP, Analyse non linéaire, 13:635-690, 1996.

[5] M.S. Aronna. Singular solutions in optimal control: second order conditions and a shooting algorithm. Preprint, 2012.

[6] M.S. Aronna, J.F. Bonnans, A.V. Dmitruk, and P.A. Lotito. Quadratic order conditions for bang-singular extremals. Numer. Algebra Contr. Optim., special issue in honor of Helmut Maurer, 2(3):511-546, 2012.

[7] L.D. Berkovitz. Variational methods in problems of control and programming. J. Math. Anal. Appl., 3:145$169,1961$.

[8] Bernard Bonnard and Monique Chyba. Singular trajectories and their role in control theory, volume 40 of Mathématiques et Applications. Springer, New York, 2012.

[9] Y. Chitour, F. Jean, and E. Trélat. Singular trajectories of control-affine systems. SIAM J. Control Optim., 47(2):1078-1095, 2008.

[10] F.H. Clarke. Optimization and nonsmooth analysis, volume 5 of Classics in Applied Mathematics. Society for Industrial and Applied Mathematics (SIAM), Philadelphia, PA, second edition, 1990.

[11] M.C. Delfour and J.-P. Zolésio. Shapes and geometries, volume 22 of Advances in Design and Control. Society for Industrial and Applied Mathematics (SIAM), Philadelphia, PA, second edition, 2011.

[12] A.V. Dmitruk. Quadratic conditions for the Pontryagin minimum in an optimal control problem that is linear with respect to control, with a constraint on the control. Dokl. Akad. Nauk SSSR, 272(2):285-289, 1983.

[13] A.V. Dmitruk. Quadratic order conditions for a Pontryagin minimum in an optimal control problem linear in the control. Math. USSR Izveztiya, 28:275-303, 1987.

[14] R. Gabasov and F.M. Kirillova. High order necessary conditions for optimality. SIAM J. Control, 10:127-168, 1972 . 
[15] B.S. Goh. Necessary conditions for singular extremals involving multiple control variables. SIAM J. Control, 4(4):716-731, 1966.

[16] B.S. Goh. The second variation for the singular Bolza problem. SIAM J. Control, 4(2):309-325, 1966.

[17] B.S. Goh. Optimal singular rocket and aircraft trajectories. CCDC 2008, pages 1531-1536, 2008.

[18] D. Hoehener. Variational approach to second-order optimality conditions for control problems with pure state constraints. SIAM J. Control, 50(3):1139-1173, 2012.

[19] A.J. Krener. The high order maximal principle. Geometric methods in Systems Theory. D.Q. Mayne and R.W. Brockett, editors, Reidel, Dordrecht, 1973.

[20] A.J. Krener. The high order maximal principle and its application to singular extremals. SIAM J. Control Optimization, 15(2):256-293, 1977.

[21] D.F. Lawden. Optimal trajectories for space navigation. Butterworths, London, 1963.

[22] Heinz Schättler and Urszula Ledzewicz. Geometric optimal control, volume 38 of Interdisciplinary Applied Mathematics. Springer, New York, 2012.

[23] R.B. Vinter. Optimal control. Birkhäuser Boston Inc., Boston, 2000.

HÉLÈNE FRANKOWSKA

Institut de Mathématiques de Jussieu, Université Pierre et Marie Curie, case 247, 4 Place Jussieu, 75252 Paris, France,

PhONe : +33144277187 FAX : +33144272724

E-mail address: frankowska@math.jussieu.fr

DANIELA TONON

Institut de Mathématiques de Jussieu, Université Pierre et Marie Curie, case 247, 4 Place Jussieu, 75252 PARIs, France,

PHONE + 33144279863

E-mail address: tonondaniela83@gmail.com 